\begin{tabular}{|c|l|}
\hline Title & $\begin{array}{l}\text { Physical subspace in a model of the quantized electromagnetic field coupled to an external field with an indefinite } \\
\text { metric. }\end{array}$ \\
\hline Author(s) & Suzuki, A kito \\
\hline Citation & Hokkaido University Preprint Series in Mathematics, 886, 1-26 \\
\hline Issue Date & 2007-12-07 \\
\hline DOI & 10.14943/84036 \\
\hline Doc URL & http://hdl.handle.net/2115/69695 \\
\hline Type & bulletin (article) \\
\hline File Information & pre886.pdf \\
\hline
\end{tabular}

Instructions for use 


\title{
Physical subspace in a model of the quantized electromagnetic field coupled to an external field with an indefinite metric.
}

\author{
Akito Suzuki * \\ Department of Mathematics, \\ Hokkaido University, \\ Sapporo 060-0810, Japan \\ e-mail: akito@math.sci.hokudai.ac.jp
}

December 7, 2007

\begin{abstract}
We study a model of the quantized electromagnetic field interacting with an external static source $\rho$ in the Feynman (Lorentz) gauge and construct the quantized radiation field $\boldsymbol{A}_{\mu}(\mu=0,1,2,3)$ as an operatorvalued distribution acting on the Fock space $\mathcal{F}$ with an indefinite metric. By using the Gupta subsidiary condition $\partial^{\mu} \boldsymbol{A}_{\mu}(x)^{(+)} \Psi=0$, one can select the physical subspace $\mathcal{V}_{\text {phys }}$. According to the Gupta-Bleuler formalism, $\mathcal{V}_{\text {phys }}$ is a non-negative subspace so that elements of $\mathcal{V}_{\text {phys }}$, called physical states, can be probabilistically interpretable. Indeed, assuming that the external source $\rho$ is infrared regular, i.e., $\hat{\rho} /|\mathbf{k}|^{3 / 2} \in L^{2}\left(\mathbb{R}^{3}\right)$, we can characterize the physical subspace $\mathcal{V}_{\text {phys }}$ and show that $\mathcal{V}_{\text {phys }}$ is non-negative. In addition, we find that the Hamiltonian of the model is reduced to the Hamiltonian of the transversal photons with the Coulomb interaction. We however prove that the physical subspace is trivial, i.e., $\mathcal{V}_{\text {phys }}=0$, if and only if the external source $\rho$ is infrared singular, i.e., $\hat{\rho} /|\mathbf{k}|^{3 / 2} \notin L^{2}\left(\mathbb{R}^{3}\right)$. We also discuss a representation different from the above representation such that the physical subspace is not trivial under the infrared singular condition.
\end{abstract}

\section{Introduction}

We consider the quantized electromagnetic field interacting with an external static source. We construct the quantized radiation field $\boldsymbol{A}_{\mu}(\mu=0,1,2,3)$ as an operator-valued distribution. It is well known that, in the Coulomb gauge, the radiation field $\boldsymbol{A}_{\mu}$ can be constructed on the usual Fock space, which is a Hilbert space with an positive definite metric $[1,9,11]$. In this paper, we construct the radiation field $\boldsymbol{A}_{\mu}$ in the Feynman (Lorentz) gauge. In this case the state space $\mathcal{F}$ where the radiation field acts is an indefinite metric space $[2,3$,

\footnotetext{
${ }^{*}$ Supported by Research Fellowships of the Japan Society for the Promotion of Science for Young Scientists.
} 
$4,5,7,8,10,12,13]$ such that the usual probabilistic interpretation is not valid. According to the Gupta-Bleuler formalism, one can select the probabilistically interpretable subspace $\mathcal{V}_{\text {phys }}$, called the physical subspace, which is non-negative subspace, from the whole state space $\mathcal{F}$. We are interested in characterizing the physical subspace $\mathcal{V}_{\text {phys }}$.

Let us start with the classical field equation

$$
\square A^{\mu}(x)=j^{\mu}(x), \quad x=(t, \mathbf{x}) \in \mathbb{R} \times \mathbb{R}^{3},
$$

where the function $j^{\mu}$ is a given classical conservation current:

$$
\partial^{\mu} j_{\mu}(x)=0 .
$$

Our first task is to construct the quantization of the classical radiation field $A_{\mu}$ satisfying the same equation as the classical field equation (1.1). In the Schrödinger picture, we write the Fourier expansion of the radiation field $A_{\mu}(\mathbf{x})$ as

$$
\begin{aligned}
& A_{j}(\mathbf{x})=\sum_{\lambda=1}^{3} \frac{1}{(2 \pi)^{3 / 2}} \int_{\mathbb{R}^{3}} \frac{d \mathbf{k}}{\sqrt{2|\mathbf{k}|}} e_{j}^{(\lambda)}(\mathbf{k})\left(a_{\lambda}(\mathbf{k}) e^{i \mathbf{k} \cdot \mathbf{x}}+a_{\lambda}^{\dagger}(\mathbf{k}) e^{-i \mathbf{k} \cdot \mathbf{x}}\right) \\
& A_{0}(\mathbf{x})=\frac{1}{(2 \pi)^{3 / 2}} \int_{\mathbb{R}^{3}} \frac{d \mathbf{k}}{\sqrt{2|\mathbf{k}|}}\left(a_{0}(\mathbf{k}) e^{i \mathbf{k} \cdot \mathbf{x}}+a_{0}^{\dagger}(\mathbf{k}) e^{-i \mathbf{k} \cdot \mathbf{x}}\right)
\end{aligned}
$$

where $e_{j}^{(\lambda)}(\mathbf{k})$ is the $j$ th component of the polarization vector with

$$
\begin{gathered}
e_{j}^{(3)}(\mathbf{k})=\frac{k_{j}}{|\mathbf{k}|}, \quad j=1,2,3, \\
\sum_{j=1}^{3} e_{j}^{(\lambda)}(\mathbf{k}) e_{j}^{\left(\lambda^{\prime}\right)}(\mathbf{k})=\delta_{\lambda \lambda^{\prime}}, \quad \lambda, \lambda^{\prime}=1,2,3 .
\end{gathered}
$$

Here we require that $a_{\mu}(\mathbf{k})$ and its adjoint $a_{\mu}^{\dagger}(\mathbf{k})$, called the annihilation and creation operators respectively, satisfy the canonical commutation relations (CCRs) as follows:

$$
\begin{aligned}
& {\left[a_{\mu}(\mathbf{k}), a_{\nu}^{\dagger}\left(\mathbf{k}^{\prime}\right)\right]=-g_{\mu \nu} \delta\left(\mathbf{k}-\mathbf{k}^{\prime}\right)} \\
& {\left[a_{\mu}(\mathbf{k}), a_{\nu}\left(\mathbf{k}^{\prime}\right)\right]=0=\left[a_{\mu}^{\dagger}(\mathbf{k}), a_{\nu}^{\dagger}\left(\mathbf{k}^{\prime}\right)\right],}
\end{aligned}
$$

where the $4 \times 4$ matrix $\left(g_{\mu \nu}\right)_{\mu, \nu=0,1,2,3}$ is the diagonal matrix with

$$
\begin{aligned}
& g_{00}=-g_{j j}=1 \quad(j, k=1,2,3), \\
& g_{\mu \nu}=0 \quad(\mu \neq \nu) .
\end{aligned}
$$

Let $\mathcal{F}$ be the state space where the operators $A_{\mu}$ act and $\langle\cdot \mid \cdot\rangle$ the metric on $\mathcal{F}$. Requiring the existence of the vacuum state $\Omega \in \mathcal{F}$ characterized by

$$
a_{\mu}(\mathbf{k}) \Omega=0, \quad\langle\Omega \mid \Omega\rangle=1,
$$

we find that the state space $\mathcal{F}$ is an indefinite metric space. Indeed, for $f \in$ $L^{2}\left(\mathbb{R}^{3}\right)$, setting $\Psi_{f}=\int_{\mathbb{R}^{3}} d \mathbf{k} f(\mathbf{k}) a_{0}^{\dagger}(\mathbf{k}) \Omega$, we have

$$
\begin{aligned}
\left\langle\Psi_{f} \mid \Psi_{f}\right\rangle & =\int_{\mathbb{R}^{3}} d \mathbf{k} \int_{\mathbb{R}^{3}} d \mathbf{q} f^{*}(\mathbf{k}) f(\mathbf{q})\left\langle\Omega \mid\left[a_{0}(\mathbf{k}), a_{0}^{\dagger}(\mathbf{q})\right] \Omega\right\rangle \\
& =-\int_{\mathbb{R}^{3}}|f(\mathbf{k})|^{2} d \mathbf{k}<0
\end{aligned}
$$


For simplicity we assume that $j^{\mu}$ is real and static:

$$
j^{0}(x)=\rho(\mathbf{x}) \in \mathbb{R}, \quad j^{i}(x)=0(i=1,2,3),
$$

where $\rho$ is the charge density and $\mathbf{j}=\left(j^{1}, j^{2}, j^{3}\right)$ the current density. Then the current $j^{\mu}$ is conserved:

$$
\partial_{\mu} j^{\mu}(x)=\frac{\partial}{\partial t} \rho(\mathbf{x})=0 .
$$

In terms of the annihilation and creation operators, the Hamiltonian of this interaction system is written as

$$
H=H_{\mathrm{f}}+H_{\text {int }},
$$

where $H_{\mathrm{f}}$ is the free Hamiltonian of the electromagnetic field of the form

$$
H_{\mathrm{f}}=\int_{\mathbb{R}^{3}}|\mathbf{k}|\left[\sum_{j=1}^{3} a_{j}^{\dagger}(\mathbf{k}) a_{j}(\mathbf{k})-a_{0}^{\dagger}(\mathbf{k}) a_{0}(\mathbf{k})\right]
$$

and $H_{\text {int }}$ the interaction Hamiltonian given by

$$
\begin{aligned}
H_{\mathrm{int}} & =\int_{\mathbb{R}^{3}} j^{\mu}(x) A_{\mu}(\mathbf{x}) d \mathbf{x} \\
& =\int_{\mathbb{R}^{3}} \frac{\mathbf{d k}}{\sqrt{2|\mathbf{k}|}}\left[\hat{\rho}(\mathbf{k})^{*} a_{0}(\mathbf{k})+\hat{\rho}(\mathbf{k}) a_{0}^{\dagger}(\mathbf{k})\right] .
\end{aligned}
$$

Here we denote the Fourier transform of $\rho$ by $\hat{\rho}$ and have used the equation $\hat{\rho}(-\mathbf{k})=\hat{\rho}(\mathbf{k})^{*}$ which is given by the assumption that $\rho$ is real. We note that $H$ is Hermite with respect to the indefinite metric $\langle\cdot \mid \cdot\rangle$ :

$$
\langle\psi \mid H \psi\rangle=\langle H \psi \mid \psi\rangle \text {. }
$$

Let us consider the initial value problem of the Schödinger equation:

$$
\begin{aligned}
i \frac{d}{d t} \psi(t) & =H \psi(t), \\
\psi(0) & =\psi \in \mathcal{F} .
\end{aligned}
$$

To this end, we require that the state space $\mathcal{F}$ has a topological structure. Let us assume that $\mathcal{F}$ is a Krein space: There exists an operator $\eta$ acting on $\mathcal{F}$ such that the following three conditions hold; (i) The metric $\langle\cdot, \cdot\rangle:=\langle\cdot \mid \eta \cdot\rangle$ is positive definite, (ii) $\mathcal{F}$ is a Hilbert space with respect to the metric $\langle\cdot, \cdot\rangle$ and (iii) $\eta=\eta^{*}=\eta^{-1}$, where ${ }^{*}$ denotes the Hilbert adjoint. By this assumption, the state space $\mathcal{F}$ has a complete norm topology by the norm $\sqrt{\langle\cdot, \cdot\rangle}$ although $\mathcal{F}$ is an indefinite metric space. Hence the derivation $\frac{d}{d t}$ in the equation (1.4) makes sense as the strong topology of the Krein space $\mathcal{F}$.

In general, if $H$ is a selfadjoint operator on a Hilbert space, i.e., $H=H^{*}$, there exists the time evolution operator $e^{-i t H}$ such that $\psi(t)=e^{-i t H} \psi$ is the unique solution of (1.4) with the initial value (1.5). We say that a densely defined linear operator $T$ is $\eta$-selfadjoint if $T=\eta T^{*} \eta$. We note that, as will be seen later, $H$ is $\eta$-selfadjoint but not selfadjoint (if $\rho \not \equiv 0$ ), i.e., $H=\eta H^{*} \eta \neq H^{*}$, 
and hence the time evolution operator $e^{-i t H}$ does not necessarily exist. This means that the existence and uniqueness of solution (1.4) with (1.5) is not trivial. It is hence difficult to discuss the time evolution of states in the Krein space.

Let us transform the field operators $A_{\mu}$ of the Schrödinger picture into the field operators $\boldsymbol{A}_{\mu}$ of the Heisenberg picture, which are called the Heisenberg operators and given by the Heisenberg equations:

$$
\begin{aligned}
\frac{d}{d t} \boldsymbol{A}_{\mu}(t, \mathbf{x}) & =i\left[H, \boldsymbol{A}_{\mu}(t, \mathbf{x})\right], \\
\boldsymbol{A}_{\mu}(0, \mathbf{x}) & =A_{\mu}(\mathbf{x}) .
\end{aligned}
$$

If $H$ is a selfadjoint operator on a Hilbert space, we obtain $\boldsymbol{A}_{\mu}$ by the usual form

$$
\boldsymbol{A}_{\mu}(t, \mathbf{x})=e^{i t H} A_{\mu}(\mathbf{x}) e^{-i t H},
$$

which, formally, satisfy the field equations

$$
\square \boldsymbol{A}_{\mu}(x)=j_{\mu}(x)
$$

with $j_{0}(x)=\rho(\mathbf{x})$ and $\mathbf{j}=0$. As we mentioned above, since $H$ is not selfadjoint if $\rho \not \equiv 0$, the right hand side of (1.8) makes no sense. It is hence not easy to transform the operators of the Schrödinger picture into those of the Heisenberg picture, differing from the usual quantum theory on a Hilbert space. If, however, there exist solutions $\boldsymbol{A}_{\mu}$ of the Heisenberg equations (1.6) with the initial values (1.7) such that $\boldsymbol{A}_{\mu}$ obey the field equations (1.9), then we can prove that the solutions $\boldsymbol{A}_{\mu}$ are unique. As will be seen later, we can construct the Heisenberg operators $\boldsymbol{A}_{\mu}$.

Let us now explain the Gupta-Bleuler formalism in our model. Formally, by (1.9) and the conservation law $\partial_{\mu} j^{\mu}=0$, we find that $\partial^{\mu} \boldsymbol{A}_{\mu}$ is a free field:

$$
\square \partial^{\mu} \boldsymbol{A}_{\mu}(x)=0 .
$$

Hence we may write $\partial^{\mu} \boldsymbol{A}_{\mu}(x)$ as

$$
\partial^{\mu} \boldsymbol{A}_{\mu}(x)=\int_{\mathbb{R}^{3}} d \mathbf{k}\left(\alpha(\mathbf{k}) e^{-i \omega(\mathbf{k}) t} e^{i \mathbf{k} \cdot \mathbf{x}}+\alpha^{\dagger}(\mathbf{k}) e^{i \omega(\mathbf{k}) t} e^{-i \mathbf{k} \cdot \mathbf{x})}\right) .
$$

In the above equation, the term which has the factor $e^{-i \omega(\mathbf{k}) t}\left(\operatorname{resp} . e^{i \omega(\mathbf{k}) t}\right)$ is called the positive frequency part (resp. negative frequency part) of $\partial^{\mu} \boldsymbol{A}_{\mu}(x)$ and written as $\left[\partial^{\mu} \boldsymbol{A}_{\mu}(x)\right]^{(+)}\left(\operatorname{resp} .\left[\partial^{\mu} \boldsymbol{A}_{\mu}(x)\right]^{(-)}\right)$. For the state vectors $\Psi \in \mathcal{F}$, the condition

$$
\left[\partial^{\mu} \boldsymbol{A}_{\mu}(t, \mathbf{x})\right]^{(+)} \Psi=0
$$

is called the Gupta subsidiary condition $[2,3,4,8,12,13]$. The physical subspace $\mathcal{V}_{\text {phys }}$ is formally defined by

$$
\mathcal{V}_{\text {phys }} \equiv\left\{\Psi \mid\left[\partial^{\mu} \boldsymbol{A}_{\mu}(x)\right]^{(+)} \Psi=0, x \in \mathbb{R}^{4}\right\}
$$

In other words, the physical subspace $\mathcal{V}_{\text {phys }}$ is the solution space of the vector equation (1.10) for all $x \in \mathbb{R}^{4}$. The zero vector $0 \in \mathcal{F}$ is a trivial solution of (1.10). We say that the physical subspace $\mathcal{V}_{\text {phys }}$ is trivial if $\mathcal{V}_{\text {phys }}=\{0\}$. It 
is expected that the physical subspace $\mathcal{V}_{\text {phys }}$ is non-trivial: $\mathcal{V}_{\text {phys }} \neq\{0\}$. It is, however, not clear whether $\mathcal{V}_{\text {phys }}$ is non-trivial or not. Indeed, we prove that $\mathcal{V}_{\text {phys }}$ is non-trivial if and only if $\rho \in H_{\omega}^{-3 / 2}\left(\mathbb{R}^{3}\right)$, where

$$
H_{\omega}^{s}\left(\mathbb{R}^{3}\right)=\left\{f \in \mathscr{S}^{\prime}\left(\mathbb{R}^{3}\right) \mid \omega^{s} \hat{f} \in L^{2}\left(\mathbb{R}^{3}\right)\right\}
$$

We say that $\rho$ is infrared regular if $\rho \in H_{\omega}^{-3 / 2}\left(\mathbb{R}^{3}\right)$ and that $\rho$ is infrared singular if $\rho \notin H_{\omega}^{-3 / 2}\left(\mathbb{R}^{3}\right)$.

Let us here mention the physical meaning of $\mathcal{V}_{\text {phys }}$ (for more details see $[6,7,8])$. Once we can show that $\mathcal{V}_{\text {phys }}$ is non-negative, i.e.,

$$
\langle\Psi \mid \Psi\rangle \geq 0, \quad \Psi \in \mathcal{V}_{\text {phys }}
$$

we obtain the physical Hilbert space $\mathcal{H}_{\text {phys }}$ given by the completion of the quotient vector space $\mathcal{V}_{\text {phys }} / \mathcal{V}_{0}$ :

$$
\mathcal{H}_{\text {phys }}=\overline{\mathcal{V}_{\text {phys }} / \mathcal{V}_{0}}
$$

where $\mathcal{V}_{0}$ is the subspace of all neutral vectors of $\mathcal{V}_{\text {phys }}$ :

$$
\mathcal{V}_{0}=\left\{\Psi \in \mathcal{V}_{\text {phys }} \mid\langle\Psi \mid \Psi\rangle=0\right\} .
$$

If the Hamiltonian $H$ leaves $\mathcal{V}_{\text {phys }}$ invariant, i.e., $H\left(\mathcal{V}_{\text {phys }} \cap D(H)\right) \subset \mathcal{V}_{\text {phys }}$, one can define the physical Hamiltonian $H_{\text {phys }}$ consistently on the physical Hilbert space $H_{\text {phys }}$. Indeed, as in shown later, the physical Hamiltonian is formally given by

$$
H_{\mathrm{phys}}=\sum_{j=1}^{2} \int_{\mathbb{R}^{3}} \omega(\mathbf{k}) a_{j}^{*}(\mathbf{k}) a_{j}(\mathbf{k}) d \mathbf{k}+\frac{1}{2} \int d \mathbf{k} \frac{|\hat{\rho}(\mathbf{k})|^{2}}{\omega(\mathbf{k})^{2}},
$$

which is selfadjoint It is remarkable that the first term of the right hand side in the above equation includes the energy of the transversal photons alone, which is the same form of the free Hamiltonian in the Coulomb gauge (see, e.g., $[1,11]$ ). Also we observe that the physical Hilbert space $H_{\text {phys }}$ is (unitarily) equivalent to the Hilbert space of the radiation field in the Coulomb gauge. Thus the longitudinal and scalar photons are confined, so that we have a probabilistically interpretable theory. We note, as is shown later, that the Coulomb potential appears from the second term of (1.11) in the point source limit. In the papers $[2,4]$, the Coulomb interaction is derived in the perturbative way. A feature of this paper is to derive the Coulomb potential in the non-perturbative way.

This paper is organized as follows. In Section 2, we construct the quantized radiation field on the Fock space. To begin with, in Subsection 2.1, we define the state space with an indefinite metric by using of the (boson) Fock space over $\oplus^{4} L^{2}\left(\mathbb{R}^{3}\right)$ and a metric operator $\eta$. We also introduce operators which play an important role and give the precise definition of the Hamiltonian. In Subsection 2.2, we construct the radiation field $\boldsymbol{A}_{\mu}$ and prove that $\boldsymbol{A}_{\mu}$ satisfies the Heisenberg equation (1.6) and the field equation (1.9). We also discuss the uniqueness of the solution of (1.6) and (1.9) with the initial value (1.7). In Subsection 2.3, by using the radiation field $\boldsymbol{A}_{\mu}$ constructed in Subsection 2.2, we formulate the Gupta subsidiary condition and give a precise definition of the physical subspace $\mathcal{V}_{\text {phys }}$. In Subsection 2.4, we characterize the physical subspace $\mathcal{V}_{\text {phys }}$ and prove that $\mathcal{V}_{\text {phys }}$ is non-trivial if and only if $\rho$ is infrared regular 
and that $\mathcal{V}_{\text {phys }}$ is non-negative. We also show that $H$ leaves $\mathcal{V}_{\text {phys }}$ invariant and give the expression (1.11) under the infrared regular condition of $\rho$. As a typical example, in Subsection 2.5, we consider the case of the electrostatic field. In this case, we obtain the Coulomb potential in the point source limit. In Section 3 , we construct the radiation field $\boldsymbol{A}_{\mu}$ by using the non-Fock representation. In this case, the physical subspace is non-trivial even if $\rho$ is infrared singular.

\section{Construction on the Fock space}

\subsection{State space and Hamiltonian}

Let $\mathcal{F}$ be the boson Fock space over $\oplus^{4} L^{2}\left(\mathbb{R}^{3}\right)$ :

$$
\mathcal{F}=\mathcal{F}\left(\oplus^{4} L^{2}\left(\mathbb{R}^{3}\right)\right)=\bigoplus_{n=0}^{\infty} \bigotimes_{\mathrm{s}}^{n}\left[\oplus^{4} L^{2}\left(\mathbb{R}^{3}\right)\right],
$$

where $\otimes_{\mathrm{s}}^{n}$ denotes the symmetric tensor product with the convention

$$
\otimes_{\mathrm{s}}^{0}\left[\oplus^{4} L^{2}\left(\mathbb{R}^{3}\right)\right]=\mathbb{C} .
$$

A vector $\Psi \in \mathcal{F}$ can be identified with the sequence $\left\{\Psi^{(n)}\right\}_{n=0}^{\infty}$ of the $4^{n}$ component squared integrable functions $\Psi^{(n)}=\left\{\Psi_{\mu_{1}, \cdots, \mu_{n}}^{(n)}\right\}_{\mu_{l}=0,1,2,3}$ satisfying that, for all permutations $\pi$,

$$
\Psi_{\mu_{1}, \cdots, \mu_{n}}^{(n)}\left(\mathbf{k}_{1}, \cdots, \mathbf{k}_{n}\right)=\Psi_{\mu_{\pi(1)}, \cdots, \mu_{\pi(n)}}^{(n)}\left(\mathbf{k}_{\pi(1)}, \cdots, \mathbf{k}_{\pi(n)}\right) .
$$

The metric of $\mathcal{F}$ is given by

$$
\begin{aligned}
&\langle\Psi \mid \Phi\rangle=\sum_{n=0}^{\infty} \sum_{\substack{\mu_{l}=0 \\
l=1, \cdots, n}}^{3} \int_{\mathbb{R}^{3 n}} \Psi_{\mu_{1}, \cdots, \mu_{n}}^{(n)}\left(\mathbf{k}_{1}, \cdots, \mathbf{k}_{n}\right)^{*} \\
& \times\left(-g_{\mu_{1} \nu_{1}}\right) \cdots\left(-g_{\mu_{n} \nu_{n}}\right) \Phi_{\nu_{1}, \cdots, \nu_{n}}^{(n)}\left(\mathbf{k}_{1}, \cdots, \mathbf{k}_{n}\right)
\end{aligned}
$$

for all $\Psi, \Phi \in \mathcal{F}$. It is clear, from $(1.2)$ and $(1.3)$, that $(\mathcal{F},\langle\cdot \mid \cdot\rangle)$ is an indefinite metric space. It is well known that the Fock space $\mathcal{F}$ is a Hilbert space with respective to a positive definite metric given by

$$
\langle\Psi, \Phi\rangle=\sum_{n=0}^{\infty} \sum_{\substack{\mu_{l}=0 \\ l=1, \cdots, n}}^{3} \int_{\mathbb{R}^{3 n}} \Psi_{\mu_{1}, \cdots, \mu_{n}}^{(n)}\left(\mathbf{k}_{1}, \cdots, \mathbf{k}_{n}\right)^{*} \Phi_{\mu_{1}, \cdots, \mu_{n}}^{(n)}\left(\mathbf{k}_{1}, \cdots, \mathbf{k}_{n}\right) .
$$

Let us define an operator $\eta$ on $\mathcal{F}$ by

$$
(\eta \Psi)_{\mu_{1}, \cdot, \mu_{n}}^{(n)}\left(\mathbf{k}_{1}, \cdots, \mathbf{k}_{n}\right)=\left(-g_{\mu_{1} \nu_{1}}\right) \cdots\left(-g_{\mu_{n} \nu_{n}}\right) \Psi_{\nu_{1}, \cdots, \nu_{n}}^{(n)}\left(\mathbf{k}_{1}, \cdots, \mathbf{k}_{n}\right), \quad \text { a.e. }
$$

We observe that $\eta=\eta^{*}=\eta^{-1}$ and that

$$
\langle\Phi \mid \Psi\rangle=\langle\Phi, \eta \Psi\rangle, \quad \Psi, \Phi \in \mathcal{F},
$$

where $^{*}$ denotes the usual Hilbert adjoint with respect to $\langle\cdot, \cdot\rangle$. Hence $\mathcal{F}$ is a Krein space with the metric operator $\eta$. We consider that the state space $\mathcal{F}$ is the Krein space $(\mathcal{F},\langle\cdot, \cdot\rangle)$ which has the norm topology given by the norm

$$
\|\Psi\|=\sqrt{\langle\Psi, \Psi\rangle}, \quad \Psi \in \mathcal{F} .
$$


For each $\Psi \in \mathcal{F}$, we define $a_{\mu}(\mathbf{k}) \Psi \in \times_{n=0}^{\infty} \otimes_{\mathrm{s}}^{n}\left[\oplus^{4} L^{2}\left(\mathbb{R}^{3}\right)\right]$ by

$$
\left(a_{\mu}(\mathbf{k}) \Psi\right)_{\mu_{1}, \cdots, \mu_{n}}^{(n)}\left(\mathbf{k}_{1}, \cdots, \mathbf{k}_{n}\right)=\sqrt{n+1} \Psi_{\mu, \mu_{1}, \cdots, \mu_{n}}^{(n+1)}\left(\mathbf{k}, \mathbf{k}_{1}, \cdots, \mathbf{k}_{n}\right), \quad \text { a.e. }
$$

where the symbol " $\times$ " denotes the Cartesian product. Let

$$
D\left(a_{\mu}(\mathbf{k})\right):=\left\{\Psi \in \mathcal{F} \mid a_{\mu}(\mathbf{p}) \Psi \in \mathcal{F}, \text { a.e.p } \in \mathbb{R}^{3}\right\} .
$$

We note that $D\left(a_{\mu}(\mathbf{k})\right)$ is independent of $\mathbf{k}$. The Fock vacuum $\Omega:=\{1,0,0, \cdots\} \in$ $\mathcal{F}$ satisfies that $\Omega \in D\left(a_{\mu}(\mathbf{k})\right)$ and that

$$
a_{\mu}(\mathbf{k}) \Omega=0
$$

Let

$$
a_{\mu}(f)=\int_{\mathbb{R}^{3}} f^{*}(\mathbf{k}) a_{\mu}(\mathbf{k}) d \mathbf{k}, \quad f \in L^{2}\left(\mathbb{R}^{3}\right) .
$$

We also denote the closure of $a(f)$ by the same symbol.

In general, the adjoint operator $A^{\dagger}$ of an operator $A$ on the Krein space $\mathcal{K}$ with the metric operator $\iota$ is given by $A^{\dagger}=\iota A^{*} \iota$. We observe that the adjoint of $a_{\mu}(f)$ is given by

$$
a_{\mu}^{\dagger}(f)= \begin{cases}-a_{0}(f)^{*}, & \mu=0 \\ a_{j}(f)^{*}, & \mu=j=1,2,3,\end{cases}
$$

and that for all $f, g \in L^{2}\left(\mathbb{R}^{3}\right)$,

$$
\begin{aligned}
& {\left[a_{\mu}(f), a_{\nu}^{\dagger}(g)\right]=-g_{\mu \nu} \int_{\mathbb{R}^{3}} f^{*}(\mathbf{k}) g(\mathbf{k}) d \mathbf{k},} \\
& {\left[a_{\mu}(f), a_{\nu}(g)\right]=\left[a_{\mu}^{\dagger}(f), a_{\nu}^{\dagger}(g)\right]=0}
\end{aligned}
$$

on the finite particle space $\mathcal{F}_{0}$ where

$$
\mathcal{F}_{0}=\left\{\Psi \in \mathcal{F} \mid \Psi^{(n)}=0 \text { for all } n \geq n_{0} \text { with some } n_{0} \in \mathbb{N}\right\}
$$

Formally, we write

$$
a_{\mu}^{\dagger}(f)=\int_{\mathbb{R}^{3}} d \mathbf{k} f(\mathbf{k}) a_{\mu}^{\dagger}(\mathbf{k})
$$

We define the free Hamiltonian of the radiation field $H_{\mathrm{f}}$ by

$$
\begin{aligned}
& D\left(H_{\mathrm{f}}\right):=\left\{\left.\Psi \in \mathcal{F}\left|\sum_{n=0}^{\infty} \sum_{\mu_{1}, \cdots, \mu_{n}} \int_{\mathbb{R}^{n}} \prod_{j=1}^{n} d \mathbf{k}_{j}\right|\left(H_{\mathrm{f}} \Psi\right)_{\mu_{1}, \cdots, \mu_{n}}^{(n)}\left(\mathbf{k}_{1}, \cdots, \mathbf{k}_{n}\right)\right|^{2}<\infty\right\}, \\
& \left(H_{\mathrm{f}} \Psi\right)_{\mu_{1}, \cdots, \mu_{n}}^{(n)}\left(\mathbf{k}_{1}, \cdots, \mathbf{k}_{n}\right)=\left(\sum_{j=1}^{n} \omega\left(\mathbf{k}_{j}\right)\right) \Psi_{\mu_{1}, \cdots, \mu_{n}}^{(n)}\left(\mathbf{k}_{1}, \cdots, \mathbf{k}_{n}\right), \quad n \geq 1
\end{aligned}
$$

and $\left(H_{\mathrm{f}} \Psi\right)^{(0)}=0$ with $\omega(\mathbf{k})=|\mathbf{k}|$. It is well-known that $H_{\mathrm{f}}$ is a selfadjoint operator, i.e. $H_{\mathrm{f}}=H_{\mathrm{f}}^{*}$, and that

$$
H_{\mathrm{f}} \Omega=0 .
$$


We observe that, for all $\Psi \in \mathcal{F}$ and $\Phi \in D\left(H_{\mathrm{f}}\right)$,

$$
\begin{aligned}
\left\langle\Psi, H_{\mathrm{f}} \Phi\right\rangle= & \sum_{n=0}^{\infty} \sum_{\mu_{1}, \cdots, \mu_{n+1}} \int_{\mathbb{R}^{(n+1)}} \prod_{j=1}^{n+1} d \mathbf{k}_{j} \Psi_{\mu_{1}, \cdots, \mu_{n+1}}^{(n+1)}\left(\mathbf{k}_{1}, \cdots, \mathbf{k}_{n+1}\right)^{*} \\
& \times\left(\sum_{j=1}^{n+1} \omega\left(\mathbf{k}_{j}\right)\right) \Psi_{\mu_{1}, \cdots, \mu_{n+1}}^{(n+1)}\left(\mathbf{k}_{1}, \cdots, \mathbf{k}_{n+1}\right) \\
= & \sum_{n=0}^{\infty} \sum_{\mu, \mu_{1}, \cdots, \mu_{n}} \int_{\mathbb{R} \times \mathbb{R}^{n}} d \mathbf{k} \prod_{j=1}^{n} d \mathbf{k}_{j}\left(a_{\mu}(\mathbf{k}) \Psi\right)_{\mu_{1}, \cdots, \mu_{n}}^{(n)}\left(\mathbf{k}_{1}, \cdots, \mathbf{k}_{n}\right)^{*} \\
& \times \omega(\mathbf{k})\left(a_{\mu}(\mathbf{k}) \Psi\right)_{\mu_{1}, \cdots, \mu_{n}}^{(n)}\left(\mathbf{k}_{1}, \cdots, \mathbf{k}_{n}\right) .
\end{aligned}
$$

Hence we have

$$
\left\langle\Psi, H_{\mathrm{f}} \Phi\right\rangle=\sum_{\mu} \int_{\mathbb{R}} d k \omega(\mathbf{k})\left\langle a_{\mu}(\mathbf{k}) \Psi, a_{\mu}(\mathbf{k}) \Phi\right\rangle
$$

and, in this sense, write symbolically

$$
H_{\mathrm{f}}=\int_{\mathbb{R}} d \mathbf{k} \omega(\mathbf{k})\left[\sum_{j=1}^{3} a_{j}^{\dagger}(\mathbf{k}) a_{j}(\mathbf{k})-a_{0}^{\dagger}(\mathbf{k}) a_{0}(\mathbf{k})\right] .
$$

In the same way as (2.1), the number operator, $N_{\mathrm{f}}$, is given by

$$
N_{\mathrm{f}}=\int_{\mathbb{R}} d \mathbf{k}\left[\sum_{j=1}^{3} a_{j}^{\dagger}(\mathbf{k}) a_{j}(\mathbf{k})-a_{0}^{\dagger}(\mathbf{k}) a_{0}(\mathbf{k})\right] .
$$

We remark that

$$
D\left(H_{\mathrm{f}}^{1 / 2}\right), D\left(N_{\mathrm{f}}^{1 / 2}\right) \subset D\left(a_{\mu}(\mathbf{k})\right),
$$

since, for all $\Psi \in D\left(H_{\mathrm{f}}^{1 / 2}\right)$ and $\Phi \in D\left(N_{\mathrm{f}}^{1 / 2}\right)$,

$$
\begin{aligned}
& \left\|H_{\mathrm{f}}^{1 / 2} \Psi\right\|^{2}=\int_{\mathbb{R}} d k \omega(k)\left\|a_{\mu}(\mathbf{k}) \Psi\right\|^{2}<\infty, \\
& \left\|N_{\mathrm{f}}^{1 / 2} \Phi\right\|^{2}=\int_{\mathbb{R}} d k\left\|a_{\mu}(\mathbf{k}) \Phi\right\|^{2}<\infty .
\end{aligned}
$$

Let us assume that $\rho \in \mathscr{S}^{\prime}\left(\mathbb{R}^{3}\right)$ is a real distribution and

$$
\rho \in H_{\omega}^{-1 / 2}\left(\mathbb{R}^{3}\right),
$$

where

$$
H_{\omega}^{s}\left(\mathbb{R}^{3}\right):=\left\{\rho \in \mathscr{S}^{\prime}\left(\mathbb{R}^{3}\right) \mid \omega^{s} \hat{\rho} \in L^{2}\left(\mathbb{R}^{3}\right)\right\}, \quad s \in \mathbb{R} .
$$

Then the Hamiltonian of a system of quantized electromagnetic field interacting with a classical source is defined by

$$
H=H_{\mathrm{f}}+H_{\text {int }},
$$


where $H_{\text {int }}$ is the interaction Hamiltonian given by

$$
H_{\mathrm{int}}=\frac{1}{\sqrt{2}}\left[a_{0}\left(\frac{\hat{\rho}}{\sqrt{\omega}}\right)+a_{0}^{\dagger}\left(\frac{\hat{\rho}}{\sqrt{\omega}}\right)\right] .
$$

It is easy to prove that $H_{\text {int }}$ is skewsymmetric, i.e. $H_{\text {int }}^{*}=-H_{\text {int }}$. By direct calculation we observe that $\eta$ leaves the domain $D\left(H_{\mathrm{f}}\right)$ invariant, i.e. $\eta D\left(H_{\mathrm{f}}\right) \subset$ $D\left(H_{\mathrm{f}}\right)$, and that, for all $\Psi \in D\left(H_{\mathrm{f}}\right)$

$$
\eta H_{\mathrm{f}} \eta \Psi=H_{\mathrm{f}} \Psi \quad \eta H_{\mathrm{int}} \eta \Psi=-H_{\mathrm{int}} \Psi .
$$

We have hence

$$
\left[\eta, H_{\mathrm{f}}\right]=\left\{\eta, H_{\mathrm{int}}\right\}=0
$$

on $D\left(H_{\mathrm{f}}\right)$, where $[X, Y]:=X Y-Y X$ and $\{X, Y\}=X Y+Y X$.

Proposition 2.1. Let

$$
\rho \in H_{\omega}^{-1 / 2}\left(\mathbb{R}^{3}\right) \cap H_{\omega}^{-1}\left(\mathbb{R}^{3}\right) .
$$

Then $H$ is $\eta$-selfadjoint, i.e. $H=H^{\dagger}$.

Proof. It is easy to see that $H=H^{\dagger}$ if $\eta H$ is selfadjoint. The selfadjointness of $\eta H$ is proved by the Kato-Rellich theorem.

\subsection{Heisenberg equations and field equations}

Through this section we assume that (2.5). For all $t \in \mathbb{R}$ and $f \in \mathscr{S}\left(\mathbb{R}^{3}\right)$, we set

$$
\begin{aligned}
& \boldsymbol{A}_{j}(t, f):=\frac{1}{\sqrt{2}} \sum_{\lambda=1}^{3}\left[a_{\lambda}\left(e_{j}^{(\lambda)} \frac{e^{i t \omega} \widehat{f^{*}}}{\sqrt{\omega}}\right)+a_{\lambda}^{\dagger}\left(e_{j}^{(\lambda)} \frac{e^{i t \omega} \hat{f}}{\sqrt{\omega}}\right)\right], \quad j=1,2,3, \\
& \boldsymbol{A}_{0}(t, f):=\frac{1}{\sqrt{2}}\left[a_{0}\left(\frac{e^{i t \omega} \widehat{f^{*}}}{\sqrt{\omega}}\right)+L\left(t, f^{*}\right)+a_{0}^{\dagger}\left(\frac{e^{i t \omega} \hat{f}}{\sqrt{\omega}}\right)+L(t, f)^{*}\right]
\end{aligned}
$$

where

$$
L(t, f)=-\frac{1}{\sqrt{2}} \int_{\mathbb{R}^{3}} \frac{d \mathbf{k}}{\omega(\mathbf{k})^{2}}\left(e^{-i t \omega(\mathbf{k})}-1\right) \hat{f}(\mathbf{k})^{*} \hat{\rho}(\mathbf{k}) .
$$

Proposition 2.2. For each $f \in \mathscr{S}\left(\mathbb{R}^{3}\right)$ and $\Psi \in D\left(N_{\mathrm{f}}^{1 / 2}\right)$, the map $\mathbb{R} \ni t \mapsto$ $\mathbf{A}_{\mu}(t, f) \Psi \in \mathcal{F}$ is $n$ times strongly differentiable and

$$
\begin{aligned}
& \frac{d^{n}}{d t^{n}} \boldsymbol{A}_{j}(t, f) \Psi=\frac{1}{\sqrt{2}} \sum_{\lambda=1}^{3}\left[a_{\lambda}\left(e_{j}^{(\lambda)}(i \omega)^{n} \frac{e^{i t \omega} \widehat{f^{*}}}{\sqrt{\omega}}\right)\right. \\
&\left.+a_{\lambda}^{\dagger}\left(e_{j}^{(\lambda)}(i \omega)^{n} \frac{e^{i t \omega} \hat{f}}{\sqrt{\omega}}\right)\right] \Psi, \quad j=1,2,3 \\
& \frac{d^{n}}{d t^{n}} \boldsymbol{A}_{0}(t, f) \Psi=\frac{1}{\sqrt{2}} {\left[a_{0}\left((i \omega)^{n} \frac{e^{i t \omega} \widehat{f^{*}}}{\sqrt{\omega}}\right)+L^{(n)}\left(t, f^{*}\right)\right.} \\
&+\left.a_{0}^{\dagger}\left((i \omega)^{n} \frac{e^{i t \omega} \hat{f}}{\sqrt{\omega}}\right)+L^{(n)}(t, f)^{*}\right] \Psi,
\end{aligned}
$$


where

$$
L^{(n)}(t, f)=-(-i)^{n} \frac{1}{\sqrt{2}} \int_{\mathbb{R}^{3}} d \mathbf{k} \omega(\mathbf{k})^{n-2} e^{-i t \omega(\mathbf{k})} \hat{f}(\mathbf{k})^{*} \hat{\rho}(\mathbf{k}) .
$$

Proof. It is evident from the following lemmas.

Lemma 2.3. For all $f, g \in L^{2}\left(\mathbb{R}^{3}\right)$ and $\Psi \in D\left(N_{\mathrm{f}}^{1 / 2}\right)$,

$$
\begin{aligned}
& \left\|a_{\mu}(f) \Psi\right\| \leq\|f\|\left\|N_{\mathrm{f}}^{1 / 2} \Psi\right\| \\
& \left\|a_{\mu}^{\dagger}(g) \Psi\right\| \leq\|g\|\left\|\left(N_{\mathrm{f}}+1\right)^{1 / 2} \Psi\right\| .
\end{aligned}
$$

Proof. (2.10) and (2.11) are well-known (see [?]) and easy to prove.

Lemma 2.4. For all $f, g \in D\left(\omega^{n}\right)$ and $\Psi \in D\left(N_{\mathrm{f}}^{1 / 2}\right)$, let

$$
F^{(k)}(t)=a\left((i \omega)^{n} e^{i t \omega} f\right)+a\left((i \omega)^{n} e^{i t \omega} g\right) \Psi, \quad 0 \leq k \leq n .
$$

Then the vector-valued function : $t \mapsto F^{(0)}(t)$ is $n$ times strongly differentiable and

$$
\frac{d^{n}}{d t^{n}} F^{(0)}(t)=F^{(n)}(t) \text {. }
$$

Proof. Let

$$
h_{\epsilon}(t, f)=e^{i t \omega}\left[\frac{\left(e^{i t \omega}-1\right) f}{\epsilon}-i \omega f\right], \quad f \in D(\omega) .
$$

Then

$$
\frac{F^{(k)}(t+\epsilon)-F^{(k)}(t)}{\epsilon}-F^{(k+1)}(t)=\left(a_{\mu}\left(h_{\epsilon}\left(t,(i \omega)^{k} f\right)\right)+a_{\mu}^{\dagger}\left(h_{\epsilon}\left(t,(i \omega)^{k} g\right)\right) \Psi\right.
$$

and, for all $u \in D(\omega)$,

$$
\left\|h_{\epsilon}(t, u)\right\| \rightarrow 0
$$

Hence, by (2.10) and (2.11),

$$
\frac{d}{d t} F^{(n)}(t)=F^{(n+1)}(t)
$$

which implies (2.12).

Definition 2.1. (operator-valued distribution) Let $\mathcal{K}$ be a Krein space with metric $\langle\cdot \mid \cdot\rangle$ and $\mathcal{L} \subset \mathcal{K}$ a dense subspace and set $d \in \mathbb{N}$. A map $\varphi: \mathscr{S}\left(\mathbb{R}^{d}\right) \ni$ $f \mapsto \varphi(f)$ is an operator-valued distribution on $\mathbb{R}^{d}$ acting on $(\mathcal{K}, \mathcal{L})$ if, for each $f \in \mathscr{S}\left(\mathbb{R}^{d}\right), \varphi(f)$ is a densely defined linear operator on $\mathcal{F}$ and satisfies the following properties: (i) For all $f \in \mathscr{S}\left(\mathbb{R}^{d}\right), \mathcal{L} \subset D(\varphi(f)) \cap D\left(\varphi(f)^{\dagger}\right)$, (ii) for all $f, g \in \mathscr{S}\left(\mathbb{R}^{d}\right)$ and $\alpha, \beta \in \mathbb{C}, \varphi(\alpha f+\beta g)=\alpha \varphi(f)+\beta \varphi(g)$ on $D\left(N_{\mathrm{f}}^{1 / 2}\right)$, and (iii) for all $\psi, \phi \in \mathcal{L}$, the map $f \mapsto\langle\psi \mid \varphi(f) \phi\rangle$ is a tempered distribution on $\mathbb{R}^{d}$. In this case we write formally

$$
\varphi(f)=\int_{\mathbb{R}^{d}} \varphi(x) f(x) d x
$$


and the symbol $\varphi(x)\left(x \in \mathbb{R}^{d}\right)$ the operator-valued distribution kernel.

For each $f \in \mathscr{S}\left(\mathbb{R}^{3}\right)$, we define operators $\boldsymbol{A}_{\mu}^{(n)}(t, f)$ by the action of the right hand side of (2.8) and (2.9). Hence

$$
\boldsymbol{A}_{\mu}^{(n)}(t, f) \Psi=\frac{d^{n}}{d t^{n}} \boldsymbol{A}_{\mu}(t, f) \Psi, \quad \Psi \in D\left(N_{\mathrm{f}}^{1 / 2}\right), n \geq 1 .
$$

We also write as

$$
\boldsymbol{A}_{\mu}(t, f)=\boldsymbol{A}_{\mu}^{(0)}(t, f), \quad \dot{\boldsymbol{A}}_{\mu}(t, f)=\boldsymbol{A}_{\mu}^{(1)}(t, f), \quad \ddot{\boldsymbol{A}}_{\mu}(t, f)=\boldsymbol{A}_{\mu}^{(2)}(t, f),
$$

and so on. Hence,

$$
\dot{\boldsymbol{A}}_{\mu}(t, f) \Psi=\frac{d}{d t} \boldsymbol{A}_{\mu}(t, f) \Psi, \quad \ddot{\boldsymbol{A}}_{\mu}(t, f) \Psi=\frac{d^{2}}{d t^{2}} \boldsymbol{A}_{\mu}(t, f) \Psi, \quad \Psi \in D\left(N_{\mathrm{f}}^{1 / 2}\right) .
$$

Theorem 2.5. (Heisenberg equations and field equations) For each $t \in \mathbb{R}$ and $n \geq 0$, the map $\mathscr{S}\left(\mathbb{R}^{3}\right) \ni f \mapsto \mathbf{A}_{\mu}^{(n)}(t, f)$ is an operator-valued distribution on $\mathbb{R}^{3}$ acting on $\left(\mathcal{F}, D\left(N_{\mathrm{f}}^{1 / 2}\right)\right)$ and the following hold:

(i) (observable) For all real-valued functions $f \in \mathscr{S}\left(\mathbb{R}^{3}\right), \boldsymbol{A}_{\mu}^{(n)}(f)(n \geq 0)$ are essentially $\eta$-selfadjoint on $\mathcal{F}_{0}$.

(ii)(equal-time commutation relation) For all $f, g \in \mathscr{S}\left(\mathbb{R}^{3}\right)$,

$$
\begin{aligned}
& {\left[\boldsymbol{A}_{\mu}(t, f), \dot{\boldsymbol{A}}_{\nu}(t, g)\right]=i g_{\mu \nu} \int_{\mathbb{R}^{3}} f(\mathbf{k}) g(\mathbf{k}) d \mathbf{k},} \\
& {\left[\boldsymbol{A}_{\mu}(t, f), \boldsymbol{A}_{\nu}(t, g)\right]=\left[\dot{\boldsymbol{A}}_{\mu}(t, f), \dot{\boldsymbol{A}}_{\nu}(t, g)\right]=0, \quad \text { on } \mathcal{F}_{0} .}
\end{aligned}
$$

(iii) (Heisenberg equations) For all $f \in \mathscr{S}\left(\mathbb{R}^{3}\right)$ and $\Psi \in \mathcal{F}_{0}$,

$$
\dot{\boldsymbol{A}}_{\mu}(t, f) \Psi=i\left[H, \boldsymbol{A}_{\mu}(t, f)\right] \Psi,
$$

(iv) (field equations) For all $f \in \mathscr{S}\left(\mathbb{R}^{3}\right)$

$$
\begin{aligned}
& \ddot{\boldsymbol{A}}_{j}(t, f)=\boldsymbol{A}_{j}(t, \Delta f), \\
& \ddot{\boldsymbol{A}}_{0}(t, f)=\boldsymbol{A}_{0}(t, \Delta f)+\int_{\mathbb{R}^{3}} \rho(\mathbf{x}) f(\mathbf{x}) d \mathbf{x} .
\end{aligned}
$$

Proof. By Proposition 2.2,

The operator-valued distribution $\mathscr{S}\left(\mathbb{R}^{3}\right) \ni f \mapsto \boldsymbol{A}_{\mu}(t, f)$ is called the quantized radiation field at time $t \in \mathbb{R}$.

By the equations (2.6), (2.7) and (2.13), we have

$$
\begin{aligned}
& \boldsymbol{A}_{j}(0, f)=\frac{1}{\sqrt{2}} \sum_{\lambda=1}^{3}\left[a_{\lambda}\left(e_{j}^{(\lambda)} \frac{\widehat{f^{*}}}{\sqrt{\omega}}\right)+a_{\lambda}^{\dagger}\left(e_{j}^{(\lambda)} \frac{\hat{f}}{\sqrt{\omega}}\right)\right], \quad j=1,2,3, \\
& \boldsymbol{A}_{0}(0, f)=\frac{1}{\sqrt{2}}\left[a_{0}\left(\frac{\widehat{f^{*}}}{\sqrt{\omega}}\right)+a_{0}^{\dagger}\left(\frac{\hat{f}}{\sqrt{\omega}}\right)\right], \\
& \dot{\boldsymbol{A}}_{j}(0, f)=\frac{1}{\sqrt{2}} \sum_{\lambda=1}^{3}\left[a_{\lambda}\left(i e_{j}^{(\lambda)} \sqrt{\omega} \widehat{f^{*}}\right)+a_{\lambda}^{\dagger}\left(i e_{j}^{(\lambda)} \sqrt{\omega} \hat{f}\right)\right], \quad j=1,2,3, \\
& \dot{\boldsymbol{A}}_{0}(0, f)=\frac{1}{\sqrt{2}}\left[a_{0}\left(i \sqrt{\omega} \widehat{f^{*}}\right)+L^{(1)}\left(0, f^{*}\right)+a_{0}^{\dagger}(i \sqrt{\omega} \hat{f})+L^{(1)}(0, f)^{*}\right],
\end{aligned}
$$


and

$$
L^{(1)}(0, f)=\frac{i}{\sqrt{2}} \int_{\mathbb{R}^{3}} \frac{d \mathbf{k}}{\omega(\mathbf{k})} \hat{f}(\mathbf{k})^{*} \hat{\rho}(\mathbf{k}) .
$$

Theorem 2.6. (uniqueness) Let $\varphi_{\mu}(t, \cdot)(t \in \mathbb{R})$ be operator-valued distributions on $\mathbb{R}^{3}$ acting on $\left(\mathcal{F}, \mathcal{F}_{0}\right)$. Assume that the following hold:

(A.1) For each $f \in \mathscr{S}\left(\mathbb{R}^{3}\right)$ and $\Psi \in \mathcal{F}_{0}$, the map $\mathbb{R} \ni t \mapsto \varphi_{\mu}(t, f) \Psi \in \mathcal{F}$ is 2 times strongly differentiable.

(A.2) For all $f \in \mathscr{S}\left(\mathbb{R}^{3}\right)$ and $\Psi \in \mathcal{F}_{0}$,

$$
\begin{aligned}
& \varphi_{\mu}(0, f) \Psi=\boldsymbol{A}_{\mu}(0, f) \Psi, \\
& \left.\frac{d}{d t} \varphi_{\mu}(t, f) \Psi\right|_{t=0}=\dot{\boldsymbol{A}}_{\mu}(0, f) \Psi .
\end{aligned}
$$

(A.3) For all $f \in \mathscr{S}\left(\mathbb{R}^{3}\right)$ and $\Psi \in \mathcal{F}_{0}$,

$$
\begin{aligned}
\frac{d^{2}}{d t^{2}} \varphi_{j}(t, f) \Psi & =\varphi_{j}(t, \Delta f) \Psi \\
\frac{d^{2}}{d t^{2}} \varphi_{0}(t, f) \Psi & =\left[\varphi_{0}(t, \Delta f)+\int_{\mathbb{R}^{3}} \rho(\mathbf{x}) f(\mathbf{x}) d \mathbf{x}\right] \Psi .
\end{aligned}
$$

Then, for all $f \in \mathscr{S}\left(\mathbb{R}^{3}\right)$ and $\Psi \in \mathcal{F}_{0}$,

$$
\varphi_{\mu}(t, f) \Psi=\boldsymbol{A}_{\mu}(t, f) \Psi .
$$

Proof. Let us fix arbitrarily $\Psi, \Phi \in \mathcal{F}_{0}$ and set

$$
C_{\mu}(t, f):=\left\langle\Phi \mid \varphi_{\mu}(t, f) \Psi\right\rangle-\left\langle\Phi \mid \boldsymbol{A}_{\mu}(t, f) \Psi\right\rangle, \quad t \in \mathbb{R}, \quad f \in \mathscr{S}\left(\mathbb{R}^{3}\right) .
$$

Then $\mathscr{S}\left(\mathbb{R}^{3}\right) \ni f \mapsto C_{\mu}(t, f)$ is a tempered distribution on $\mathbb{R}^{3}$ and satisfy

$$
\frac{d^{2}}{d t^{2}} C_{\mu}(t, f)=C_{\mu}(t, \Delta f)
$$

with

$$
C_{\mu}(0, f)=0,\left.\quad \frac{d}{d t} C_{\mu}(t, f)\right|_{t=0}=0
$$

Hence we find

$$
C_{\mu}(t, f)=0, \quad t \in \mathbb{R}, \quad f \in \mathscr{S}\left(\mathbb{R}^{3}\right) .
$$

Since $\Phi \in \mathcal{F}_{0}$ is chosen arbitrarily and $\mathcal{F}_{0}$ is dense in $\mathcal{F}$, we have (2.20).

Corollary 2.7. Let $\varphi_{\mu}(t, \cdot)(t \in \mathbb{R})$ be operator-valued distributions on $\mathbb{R}^{3}$ acting on $\left(\mathcal{F}, \mathcal{F}_{0}\right)$. Assume that the conditions (A.1), (A.3) in Theorem 2.6 and the following (A.2)' hold:

(A.2)' For all $f \in \mathscr{S}\left(\mathbb{R}^{3}\right)$ and $\Psi \in \mathcal{F}_{0}$,

$$
\begin{aligned}
& \frac{d}{d t} \varphi_{\mu}(t, f) \Psi=i\left[H, \varphi_{\mu}(t, f)\right] \Psi, \\
& \varphi_{\mu}(0, f) \Psi=\boldsymbol{A}_{\mu}(0, f) \Psi .
\end{aligned}
$$

Then, for all $f \in \mathscr{S}\left(\mathbb{R}^{3}\right)$ and $\Psi \in \mathcal{F}_{0},(2.20)$ holds. 
Proof. Since the condition (A.2)' implies the condition (A.2), the same conclusion as Theorem 2.6 follows.

Remark 2.1. By corollary 2.7, we conclude that the solution $\boldsymbol{A}_{\mu}(\mathbf{x})$ of the Heisenberg equations (1.6) with the initial value (1.7) satisfying the field equations (1.9) is unique in the operator-valued distribution sense.

\subsection{Physical subspace and the Gupta subsidiary condition}

For each $f \in \mathscr{S}\left(\mathbb{R}^{4}\right)$, we define an operator $\boldsymbol{A}_{\mu}(f)$ by

$$
\begin{aligned}
& \boldsymbol{A}_{j}(f):=\frac{1}{\sqrt{2}} \sum_{\lambda=1}^{3}\left[a_{\lambda}\left(e_{j}^{(\lambda)} \frac{F\left(f^{*}\right)}{\sqrt{\omega}}\right)+a_{\lambda}^{\dagger}\left(e_{j}^{(\lambda)} \frac{F(f)}{\sqrt{\omega}}\right)\right], \quad j=1,2,3, \\
& \boldsymbol{A}_{0}(f):=\frac{1}{\sqrt{2}}\left[a_{0}\left(\frac{F\left(f^{*}\right)}{\sqrt{\omega}}\right)+L\left(f^{*}\right)+a_{0}^{\dagger}\left(\frac{F(f)}{\sqrt{\omega}}\right)+L(f)^{*}\right]
\end{aligned}
$$

where

$$
\begin{aligned}
& L(f)=-\frac{1}{\sqrt{2}} \int_{\mathbb{R}^{4}} \frac{d t d \mathbf{k}}{\omega(\mathbf{k})^{2}}\left(e^{-i t \omega(\mathbf{k})}-1\right) \hat{f}(t, \mathbf{k})^{*} \hat{\rho}(\mathbf{k}), \\
& F(f)(\mathbf{k})=\int_{\mathbb{R}} e^{i t \omega(\mathbf{k})} \hat{f}(t, \mathbf{k}) d t, \quad \text { a.e. } \mathbf{k} \in \mathbb{R}^{3}
\end{aligned}
$$

with

$$
\hat{f}(t, \mathbf{k})=\frac{1}{(2 \pi)^{3 / 2}} \int_{\mathbb{R}^{3}} e^{-i \mathbf{k} \cdot \mathbf{x}} f(t, \mathbf{x}) d \mathbf{x}, \quad \text { a.e.k } \in \mathbb{R}^{3} .
$$

Proposition 2.8. The map $\mathscr{S}\left(\mathbb{R}^{4}\right) \ni f \mapsto \boldsymbol{A}_{\mu}(f)$ is an operator-valued distribution on $\mathbb{R}^{4}$ acting on $\left(\mathcal{F}, D\left(N_{\mathrm{f}}^{1 / 2}\right)\right)$ with

$$
\boldsymbol{A}_{\mu}(h \times g) \Psi=\int_{\mathbb{R}} h(t) \boldsymbol{A}_{\mu}(t, g) \Psi d t, \quad h \in \mathscr{S}(\mathbb{R}), g \in \mathscr{S}\left(\mathbb{R}^{3}\right) .
$$

Moreover, if there exists an operator-valued distribution $\varphi_{\mu}$ on $\mathbb{R}^{4}$ acting on $\left(\mathcal{F}, D\left(N_{\mathrm{f}}^{1 / 2}\right)\right.$ satisfying that

$$
\varphi_{\mu}(h \times g) \Psi=\int_{\mathbb{R}} h(t) \boldsymbol{A}_{\mu}(t, g) \Psi d t, \quad h \in \mathscr{S}(\mathbb{R}), g \in \mathscr{S}\left(\mathbb{R}^{3}\right),
$$

then

$$
\boldsymbol{A}_{\mu}(f) \Psi=\varphi(f) \Psi, \quad f \in \mathscr{S}\left(\mathbb{R}^{4}\right), \quad \Psi \in D\left(N_{\mathrm{f}}^{1 / 2}\right) .
$$

Proof. If there exists an operator-valued distribution $\mathrm{D}_{\mu}$ on $\mathbb{R}^{4}$ acting on $\left(\mathcal{F}, D\left(N_{\mathrm{f}}^{1 / 2}\right)\right.$ with then

We call the operator-valued distribution $\mathscr{S}\left(\mathbb{R}^{4}\right) \ni f \mapsto \boldsymbol{A}_{\mu}$ the quantized radiation field smeared with $f \in \mathscr{S}\left(\mathbb{R}^{4}\right)$ and denote the closure of $\boldsymbol{A}_{\mu}(f)$ by the same symbol. 
Theorem 2.9. For all $f \in \mathscr{S}\left(\mathbb{R}^{4}\right)$ and $\Psi \in D\left(N_{\mathrm{f}}^{1 / 2}\right)$, the following equations hold:

$$
\begin{gathered}
\boldsymbol{A}_{j}(\square f) \Psi=0, \quad \boldsymbol{A}_{j}(\square f) \Psi=\int_{\mathbb{R}^{4}} \rho(\mathbf{x}) d \mathbf{x} f(\mathbf{x}) \Psi, \\
\boldsymbol{A}_{\mu}\left(\partial^{\mu} f\right) \Psi=\frac{1}{\sqrt{2}}\left[a_{0}\left(i \sqrt{\omega} F\left(f^{*}\right)\right)-a_{3}\left(i \sqrt{\omega} F\left(f^{*}\right)\right)+\frac{i}{\sqrt{2}} \int_{\mathbb{R}^{3}} \frac{F\left(f^{*}\right)(\mathbf{k})^{*} \hat{\rho}(\mathbf{k})}{\omega(\mathbf{k})} d \mathbf{k}\right. \\
\left.+a_{0}^{\dagger}(i \sqrt{\omega} F(f))-a_{3}^{\dagger}(i \sqrt{\omega} F(f))-\frac{i}{\sqrt{2}} \int_{\mathbb{R}^{3}} \frac{\hat{\rho}(\mathbf{k})^{*} F(f)(\mathbf{k})}{\omega(\mathbf{k})} d \mathbf{k}\right] \Psi .
\end{gathered}
$$

Let us formulate the Gupta subsidiary condition as follows: We define the positive frequency part $\left[\partial^{\mu} \boldsymbol{A}_{\mu}(f)\right]^{(+)}$of $\partial^{\mu} \boldsymbol{A}_{\mu}(f)\left(f \in \mathscr{S}\left(\mathbb{R}^{4}\right)\right)$ by the closure of the operator

$$
\frac{i}{\sqrt{2}}\left[a_{0}\left(\sqrt{\omega} F\left(f^{*}\right)\right)-a_{3}\left(\sqrt{\omega} F\left(f^{*}\right)\right)-\frac{1}{\sqrt{2}} \int_{\mathbb{R}^{3}} \frac{F\left(f^{*}\right)(\mathbf{k})^{*} \hat{\rho}(\mathbf{k})}{\omega(\mathbf{k})} d \mathbf{k}\right]
$$

and call the following condition for vectors $\Psi \in \mathcal{F}$ the Gupta subsidiary condition

$$
\left[\partial^{\mu} \boldsymbol{A}_{\mu}(f)\right]^{(+)} \Psi=0, \quad f \in \mathscr{S}\left(\mathbb{R}^{4}\right) .
$$

Remark 2.2. In the operator-valued distribution sense, $\partial^{\mu} \boldsymbol{A}_{\mu}(f)=-\boldsymbol{A}_{\mu}\left(\partial^{\mu} f\right)$. By (2.24), we observe, formally, that

$$
\begin{array}{r}
\partial^{\mu} \boldsymbol{A}_{\mu}(x)=i \int_{\mathbb{R}^{3}} d \mathbf{k} \sqrt{\frac{\omega(\mathbf{k})}{2(2 \pi)^{3}}}\left[e^{-i \omega(\mathbf{k}) t+i \mathbf{k} \cdot \mathbf{x}}\left(a_{0}(\mathbf{k})-a_{3}(\mathbf{k})-\frac{\hat{\rho}(\mathbf{k})}{\omega(\mathbf{k})^{3 / 2}}\right)\right. \\
\left.+e^{i \omega(\mathbf{k}) t-i \mathbf{k} \cdot \mathbf{x}}\left(a_{0}^{\dagger}(\mathbf{k})-a_{3}^{\dagger}(\mathbf{k})-\frac{\hat{\rho}(\mathbf{k})}{\omega(\mathbf{k})^{3 / 2}}\right)\right] .
\end{array}
$$

The positive frequency part $\left[\partial^{\mu} \boldsymbol{A}(x)\right]^{(+)}$of (2.27) is given by the term multiplied by $e^{-i \omega(\mathbf{k}) t}$ :

$$
\left[\partial^{\mu} \boldsymbol{A}(x)\right]^{(+)}=i \int_{\mathbb{R}^{3}} d \mathbf{k} \sqrt{\frac{\omega(\mathbf{k})}{2(2 \pi)^{3}}}\left[e^{-i \omega(\mathbf{k}) t+i \mathbf{k} \cdot \mathbf{x}}\left(a_{0}(\mathbf{k})-a_{3}(\mathbf{k})-\frac{\hat{\rho}(\mathbf{k})}{\omega(\mathbf{k})^{3 / 2}}\right),\right.
$$

which is the formal expression of (2.25).

We define the physical state space $\mathcal{V}_{\text {phys }}$ by

$$
\mathcal{V}_{\text {phys }}:=\left\{\Psi \in \mathcal{F} \mid\left[\partial^{\mu} \boldsymbol{A}_{\mu}(f)\right]^{(+)} \Psi=0, \quad f \in \mathscr{S}\left(\mathbb{R}^{4}\right)\right\} .
$$

Lemma 2.10. $\mathcal{V}_{\text {phys }}$ is a closed subspace.

Proof. We observe, by definition, that

$$
\mathcal{V}_{\text {phys }}=\bigcap_{f \in \mathscr{S}\left(\mathbb{R}^{4}\right)} \operatorname{ker}\left(\left[\partial^{\mu} \boldsymbol{A}_{\mu}(f)\right]^{(+)}\right) .
$$

By the closedness of the operators $\left[\partial^{\mu} \boldsymbol{A}(f)\right]^{(+)}, \operatorname{ker}\left[\partial^{\mu} \boldsymbol{A}_{\mu}(f)\right]^{(+)}$is closed and so is $\mathcal{V}_{\text {phys }}$. 
It is easy to see, by (2.25), that a vector $\Psi \in \mathcal{F}$ satisfies the condition $(2.26)$ if and only if

$$
\left[\overline{a_{3}(u)-a_{0}(u)}+\frac{1}{\sqrt{2}} \int_{\mathbb{R}^{3}} \frac{u(\mathbf{k})^{*} \hat{\rho}(\mathbf{k})}{\omega(\mathbf{k})^{3 / 2}} d \mathbf{k}\right] \Psi=0, \quad u \in D,
$$

where $\overline{a_{3}(u)-a_{0}(u)}$ denotes the closure of $a_{3}(u)-a_{0}(u)$ and

$$
D=\left\{\sqrt{\omega} F(f) \mid f \in \mathscr{S}\left(\mathbb{R}^{4}\right)\right\} .
$$

Lemma 2.11. The subspace $D$ is dense in $L^{2}\left(\mathbb{R}^{3}\right)$.

Proof. By definition,

$$
\begin{aligned}
F(f)(\mathbf{k}) & =\frac{1}{(2 \pi)^{3 / 2}} \int_{\mathbb{R}^{4}} d t d \mathbf{x} e^{i \omega(\mathbf{k}) t-i \mathbf{k} \cdot \mathbf{x}} f(t, \mathbf{x}) \\
& =(2 \pi)^{1 / 2}(\mathscr{F} f)(-\omega(\mathbf{k}), \mathbf{k}), \quad \mathbf{k} \in \mathbb{R}^{3},
\end{aligned}
$$

where $\mathscr{F} f$ denotes the Fourier transform of $f \in \mathscr{S}\left(\mathbb{R}^{4}\right)$. Since $\mathscr{F} f$ is an element of $\mathscr{S}\left(\mathbb{R}^{4}\right)$, for all $n \geq 0$,

$$
\omega(\mathbf{k})^{n}|\mathscr{F} f(-\omega(\mathbf{k}), \mathbf{k})| \rightarrow 0, \quad|\mathbf{k}| \rightarrow \infty,
$$

which implies that, for all $f \in \mathscr{S}\left(\mathbb{R}^{4}\right), \sqrt{\omega} F(f) \in L^{2}\left(\mathbb{R}^{3}\right): D \subset L^{2}\left(\mathbb{R}^{3}\right)$. Indeed,

$$
\begin{aligned}
& \int_{\mathbb{R}^{3}} \omega(\mathbf{k})|\mathscr{F} f(-\omega(\mathbf{k}), \mathbf{k})|^{2} d \mathbf{k} \\
& =\int_{|\mathbf{k}| \leq R} \omega(\mathbf{k})|\mathscr{F} f(-\omega(\mathbf{k}), \mathbf{k})|^{2} d \mathbf{k}+\int_{|\mathbf{k}| \geq R} \omega(\mathbf{k})^{n}|\mathscr{F} f(-\omega(\mathbf{k}), \mathbf{k})|^{2} \frac{d \mathbf{k}}{\omega(\mathbf{k})^{n-1}} \\
& \leq R \int_{\mathbb{R}^{3}}|\mathscr{F} f(-\omega(\mathbf{k}), \mathbf{k})|^{2} d \mathbf{k}+\sup _{\mathbf{k} \in \mathbb{R}^{3}}\left(\omega(\mathbf{k})^{n+1}|\mathscr{F} f(-\omega(\mathbf{k}), \mathbf{k})|^{2}\right) \int_{|\mathbf{k}| \geq R} \frac{d \mathbf{k}}{\omega(\mathbf{k})^{n}} \\
& <\infty
\end{aligned}
$$

for $R>0$ with $n$ sufficiently large.

Let us arbitrarily fix $v \in C_{0}^{\infty}(\mathbb{R})$ and $w \in C_{0}^{\infty}\left(\mathbb{R}^{3}\right)$ and set $f=\hat{v} \times \check{w} \in$ $\mathscr{S}\left(\mathbb{R}^{4}\right)$ where $\hat{v}$ denotes the Fourier transform of $v$ and $\check{w}$ the inverse Fourier transform of $w$. Then we have $\sqrt{\omega} F(f)=\sqrt{\omega} v(\omega) w \in D$. Assuming that $g \in D^{\perp}$ we have

$$
\int_{\mathbb{R}^{3}} g^{*}(\mathbf{k}) \sqrt{\omega(\mathbf{k})} v(\omega(\mathbf{k})) w(\mathbf{k}) d \mathbf{k}=0 .
$$

By du Bois-Reymond lemma we observe that

$$
g^{*}(\mathbf{k}) \sqrt{\omega(\mathbf{k})} v(\omega(\mathbf{k}))=0, \quad \text { a.e.k } \in \mathbb{R}^{3},
$$

which implies that $g=0$ in $L^{2}\left(\mathbb{R}^{3}\right)$.

\subsection{Characterization of the physical subspace}

Let us now characterize the physical subspace $\mathcal{V}_{\text {phys }}$. To this end, we solve the vector equation (2.28). The following lemma is well-known and easy to see. It is the key to solving (2.28). 
Lemma 2.12. Let $\mathcal{F}(\mathcal{H})$ be the boson Fock space over a Hilbert space $\mathcal{H}, \mathcal{D} \subset$ $\mathcal{F}(\mathcal{H})$ a dense subspace in $\mathcal{H}$ and $a_{\mathcal{H}}(f)(f \in \mathcal{H})$ the annihilation operators on $\mathcal{F}(\mathcal{H})$. Then

$$
\left\{\Psi \in \mathcal{F}(\mathcal{H}) \mid a_{\mathcal{H}}(f) \Psi=0, \quad f \in \mathcal{D}\right\}=\left\{\alpha \Omega_{\mathcal{H}} \mid \alpha \in \mathbb{C}\right\},
$$

where we denote by $\Omega_{\mathcal{H}}$ the Fock vacuum of $\mathcal{F}(\mathcal{H})$ :

$$
\Omega_{\mathcal{H}}:=\{1,0,0, \cdots\} \in \mathcal{F}(\mathcal{H}) .
$$

(For the definitions of $\mathcal{F}(\mathcal{H})$ and $a_{\mathcal{H}}(f)$, see, e.g., [1].)

We use the notations of the above lemma from now on. It is convenient to introduce the following identification:

$$
\begin{aligned}
\mathcal{F} & =\mathcal{F}\left(\oplus^{4} L^{2}\left(\mathbb{R}^{3}\right)\right) \sim \mathcal{F}\left(\oplus^{3} L^{2}\left(\mathbb{R}^{3}\right)\right) \otimes \mathcal{F}\left(L^{2}\left(\mathbb{R}^{3}\right)\right) \\
& \sim \mathcal{F}\left(L^{2}\left(\mathbb{R}^{3}\right)\right) \otimes \mathcal{F}\left(L^{2}\left(\mathbb{R}^{3}\right)\right) \otimes \mathcal{F}\left(L^{2}\left(\mathbb{R}^{3}\right)\right) \otimes \mathcal{F}\left(L^{2}\left(\mathbb{R}^{3}\right)\right) .
\end{aligned}
$$

In these identifications, we recognize that

$$
\begin{aligned}
& a_{1}\left(f_{1}\right)+a_{2}\left(f_{2}\right)+a_{3}\left(f_{3}\right)+a_{0}\left(f_{0}\right)=a_{\oplus^{4} L^{2}\left(\mathbb{R}^{3}\right)}\left(f_{1}, f_{2}, f_{3}, f_{0}\right) \\
& \sim a_{\oplus^{3} L^{2}\left(\mathbb{R}^{3}\right)}\left(f_{1}, f_{2}, f_{3}\right) \otimes I+I \otimes a_{L^{2}\left(\mathbb{R}^{3}\right)}\left(f_{0}\right) \\
& \sim a_{L^{2}\left(\mathbb{R}^{3}\right)}\left(f_{1}\right) \otimes I \otimes I \otimes I+I \otimes a_{L^{2}\left(\mathbb{R}^{3}\right)}\left(f_{2}\right) \otimes I \otimes I \\
& \quad+I \otimes I \otimes a_{L^{2}\left(\mathbb{R}^{3}\right)}\left(f_{3}\right) \otimes I+I \otimes I \otimes I \otimes a_{L^{2}\left(\mathbb{R}^{3}\right)}\left(f_{0}\right)
\end{aligned}
$$

on $\mathcal{F}_{0}$, and so on.

We define a subspace $\mathcal{F}_{3}$ in the identification (2.29) by

$$
\mathcal{F}_{3}:=\mathcal{F}\left(\oplus^{3} L^{2}\left(\mathbb{R}^{3}\right)\right) \otimes\left\{\alpha \Omega_{L^{2}\left(\mathbb{R}^{3}\right)} \mid \alpha \in \mathbb{C}\right\} .
$$

We define a unitary operator $W$ by the relations

$$
W a_{\oplus^{4} L^{2}\left(\mathbb{R}^{3}\right)}\left(f_{1}, f_{2}, f_{3}, f_{0}\right) W=a_{\oplus^{4} L^{2}\left(\mathbb{R}^{3}\right)}\left(f_{1}, f_{2}, \frac{f_{3}+f_{0}}{\sqrt{2}}, \frac{f_{3}-f_{0}}{\sqrt{2}}\right)
$$

with $W \Omega=\Omega$.

For each $f \in L^{2}\left(\mathbb{R}^{3}\right), \phi_{\mu}(f)$ denotes the closure of the operator

$$
\frac{1}{\sqrt{2}}\left[a_{\mu}(f)+a_{\mu}^{*}(f)\right] .
$$

It is well known that $\phi_{\mu}(f)$ is a selfadjoint operator: $\phi_{\mu}(f)=\phi_{\mu}(f)^{*}$. If $\rho \in$ $H_{\omega}^{-3 / 2}\left(\mathbb{R}^{3}\right)$, then one can define the unitary operator $e^{-i \phi_{3}\left(i \hat{\rho} / \omega^{3 / 2}\right)}$ generated by $\phi_{3}\left(i \hat{\rho} / \omega^{3 / 2}\right)$. Let us define a unitary operator $U_{\rho}$ by

$$
U_{\rho}=W e^{-i \phi_{3}\left(i \hat{\rho} / \omega^{3 / 2}\right)} .
$$

Lemma 2.13. Let $\rho \in H_{\omega}^{-3 / 2}\left(\mathbb{R}^{3}\right)$. Then, for all $\left(f_{1}, f_{2}, f_{3}, f_{0}\right) \in \oplus^{4} L^{2}\left(\mathbb{R}^{3}\right)$,

$$
\begin{gathered}
U_{\rho} a_{\oplus^{4} L^{2}\left(\mathbb{R}^{3}\right)}\left(f_{1}, f_{2}, f_{3}, f_{0}\right) U_{\rho}^{*}=a_{\oplus^{4} L^{2}\left(\mathbb{R}^{3}\right)}\left(f_{1}, f_{2}, \frac{f_{3}+f_{0}}{\sqrt{2}}, \frac{f_{3}-f_{0}}{\sqrt{2}}\right) \\
-\frac{1}{\sqrt{2}} \int_{\mathbb{R}^{3}} \frac{f_{3}(\mathbf{k})^{*} \hat{\rho}(\mathbf{k})}{\omega(\mathbf{k})^{3 / 2}} d \mathbf{k} .
\end{gathered}
$$


Theorem 2.14. Let $\rho \in H_{\omega}^{-1 / 2}\left(\mathbb{R}^{3}\right) \cap H_{\omega}^{-3 / 2}\left(\mathbb{R}^{3}\right)$. Then

$$
\mathcal{V}_{\text {phys }}=U_{\rho}^{*} \mathcal{F}_{3}
$$

and $\mathcal{V}_{\text {phys }}$ is non-negative.

Proof. By Lemma 2.13, we observe that

$U_{\rho}\left[a_{\oplus^{4} L^{2}\left(\mathbb{R}^{3}\right)}(0,0, u,-u)+\frac{1}{\sqrt{2}} \int_{\mathbb{R}^{3}} \frac{u(\mathbf{k})^{*} \hat{\rho}(\mathbf{k})}{\omega(\mathbf{k})^{3 / 2}} d \mathbf{k}\right] U_{\rho}^{*}=\sqrt{2} a_{\oplus^{4} L^{2}\left(\mathbb{R}^{3}\right)}(0,0,0, u)$.

Hence, by (2.28), $\Psi \in \mathcal{V}_{\text {phys }}$ if and only if

$$
\sqrt{2} U_{\rho}^{*} a_{\oplus^{4} L^{2}\left(\mathbb{R}^{3}\right)}(0,0,0, u) U_{\rho} \Psi=0, \quad u \in D .
$$

By the unitarity of $U_{\rho}$ and the identification $(2.29),(2.4)$ is equivalent to the condition

$$
I \otimes a_{L^{2}\left(\mathbb{R}^{3}\right)}(u) U_{\rho} \Psi=0, \quad u \in D .
$$

Thus, by Lemma (2.12), we have

$$
U_{\rho} \mathcal{V}_{\text {phys }}=\mathcal{F}_{3}
$$

which implies (2.34).

Let us now prove the latter half of theorem. We set

$$
\mathcal{F}_{2}=\mathcal{F}\left(L^{2}\left(\mathbb{R}^{3}\right)\right) \otimes \mathcal{F}\left(L^{2}\left(\mathbb{R}^{3}\right)\right) \otimes\left\{\alpha \Omega_{L^{2}\left(\mathbb{R}^{3}\right)} \mid \alpha \in \mathbb{C}\right\} \otimes\left\{\alpha \Omega_{L^{2}\left(\mathbb{R}^{3}\right)} \mid \alpha \in \mathbb{C}\right\}
$$

in the identification (2.30). We observe that

$$
\mathcal{F}_{3}=\mathcal{F}_{2} \oplus\left[\mathcal{F}_{2}^{\perp} \cap \mathcal{F}_{3}\right]
$$

where we denote by $\mathcal{M}^{\perp}$ the (Hilbert) orthogonal complement of a set $\mathcal{M}$.

For all $\Psi \in \mathcal{V}_{\text {phys }}$, there exists a $\Phi \in \mathcal{F}_{3}$ and we can uniquely write

$$
\Phi=\Phi_{0}+\Phi_{\geq 1},
$$

where

$$
\Phi_{0} \in \mathcal{F}_{2}, \quad \Phi_{\geq 1} \in \mathcal{F}_{2}^{\perp} \cap \mathcal{F}_{3} .
$$

By the definitions of $U_{\rho}$ and $\eta$, we observe that

$$
U_{\rho}^{*} \Phi_{0}=\Phi_{0}, \quad \eta \Phi_{0}=\Phi_{0} .
$$

Hence we have

$$
\begin{aligned}
\langle\Psi \mid \Psi\rangle & =\left\langle U_{\rho}^{*}\left(\Phi_{0}+\Phi_{\geq 1}\right), \eta U_{\rho}^{*}\left(\Phi_{0}+\Phi_{\geq 1}\right)\right\rangle \\
& =\left\langle\Psi_{0}, \Psi_{0}\right\rangle+\left\langle\Phi_{\geq 1}, \Phi_{0}\right\rangle+\left\langle\Phi_{0}, \Phi_{\geq 1}\right\rangle+\left\langle U_{\rho}^{*} \Phi_{\geq 1}, \eta U_{\rho}^{*} \Phi_{\geq 1}\right\rangle \\
& =\left\langle\Psi_{0}, \Psi_{0}\right\rangle+\left\langle U_{\rho}^{*} \Phi_{\geq 1}, \eta U_{\rho}^{*} \Phi_{\geq 1}\right\rangle .
\end{aligned}
$$

The first term in the right hand side of (2.36) is non-negative.

Let us show that the second term of (2.36) vanishes. We set

$$
\mathcal{F}_{3, \mathrm{fin}}(D)=\mathscr{L}\left\{a_{j_{1}}^{\dagger}\left(u_{1}\right) \cdots a_{j_{n}}^{\dagger}\left(u_{n}\right) \Omega \mid u_{j_{i}} \in D, \quad j_{i}-1,2,3, \quad n \geq 0\right\}
$$


where we denote by $\mathscr{L} \mathcal{M}$ the linear span of a set $\mathcal{M}$. We observe that $\mathcal{F}_{3 \text {,fin }}(D)$ is dense in $\mathcal{F}_{3}$. Hence there exists a sequence $\left\{\Phi_{\geq 1, n}\right\}_{n} \subset \mathcal{F}_{3 \text {,fin }}(D)$ of the form

$$
\Phi_{\geq 1, n}=\sum_{k} a_{3}^{\dagger}\left(u_{k}^{(n)}\right) \phi_{k}^{(n)}, \quad u_{k}^{(n)} \in D \backslash\{0\}, \quad \phi_{k}^{(n)} \in \mathcal{F}_{3, \mathrm{fin}}
$$

such that $\Phi_{\geq 1, n} \rightarrow \Phi_{\geq 1}(n \rightarrow \infty)$. By (2.33), we observe that

$$
a_{\oplus^{4} L^{2}\left(\mathbb{R}^{3}\right)}(0,0, u, 0) U_{\rho}=\frac{1}{\sqrt{2}} U_{\rho}\left[a_{\oplus^{4} L^{2}\left(\mathbb{R}^{3}\right)}(0,0, u, u)+\frac{1}{\sqrt{2}} \int_{\mathbb{R}^{3}} \frac{u(\mathbf{k})^{*} \hat{\rho}(\mathbf{k})}{\omega(\mathbf{k})^{3 / 2}} d \mathbf{k}\right],
$$

which implies that

$U_{\rho}^{*} a_{\oplus^{4} L^{2}\left(\mathbb{R}^{3}\right)}(0,0, u, 0)^{*}=\frac{1}{\sqrt{2}}\left[a_{\oplus^{4} L^{2}\left(\mathbb{R}^{3}\right)}(0,0, u, u)^{*}+\frac{1}{\sqrt{2}} \int_{\mathbb{R}^{3}} \frac{u(\mathbf{k}) \hat{\rho}(\mathbf{k})^{*}}{\omega(\mathbf{k})^{3 / 2}} d \mathbf{k}\right] U_{\rho}^{*}$.

Hence, by (2.35), (2.37) and (2.38), we have

$$
\begin{aligned}
& \left\langle U_{\rho}^{*} \Phi_{\geq 1}, \eta U_{\rho}^{*} \Phi_{\geq 1}\right\rangle=\lim _{n \rightarrow \infty} \sum_{k}\left\langle U_{\rho}^{*} \Phi_{\geq 1}, \eta U_{\rho}^{*} a_{3}^{\dagger}\left(u_{k}^{(n)}\right) \phi_{k}^{(n)}\right\rangle \\
& =\lim _{n \rightarrow \infty} \frac{1}{\sqrt{2}} \sum_{k}\left\langle U_{\rho}^{*} \Phi_{\geq 1}, \eta\left[a_{3}^{\dagger}\left(u_{k}^{(n)}\right)-a_{0}\left(u_{k}^{(n)}\right)^{\dagger}+\frac{1}{\sqrt{2}} \int_{\mathbb{R}^{3}} \frac{u_{k}^{(n)}(\mathbf{k}) \hat{\rho}(\mathbf{k})^{*}}{\omega(\mathbf{k})^{3 / 2}} d \mathbf{k}\right] U_{\rho}^{*} \phi_{k}^{(n)}\right\rangle \\
& =\lim _{n \rightarrow \infty} \frac{1}{\sqrt{2}} \sum_{k}\left\langle\left[a_{3}\left(u_{k}^{(n)}\right)-a_{0}\left(u_{k}^{(n)}\right)+\frac{1}{\sqrt{2}} \int_{\mathbb{R}^{3}} \frac{u_{k}^{(n)}(\mathbf{k})^{*} \hat{\rho}(\mathbf{k})}{\omega(\mathbf{k})^{3 / 2}} d \mathbf{k}\right] U_{\rho}^{*} \Phi_{\geq 1} \mid U_{\rho}^{*} \phi_{k}^{(n)}\right\rangle \\
& =\lim _{n \rightarrow \infty} \frac{1}{\sqrt{2}} \sum_{k}\left\langle U_{\rho}^{*} a_{3}\left(u_{k}^{(n)}\right) \Phi_{\geq 1} \mid U_{\rho}^{*} \phi_{k}^{(n)}\right\rangle .
\end{aligned}
$$

We observe, by $\Phi_{\geq 1} \in \mathcal{F}_{3}$, that $a_{3}\left(u_{k}^{(n)}\right) \Phi_{\geq 1}=0$ and hence, by (2.39), that

$$
\left\langle U_{\rho}^{*} \Phi_{\geq 1}, \eta U_{\rho}^{*} \Phi_{\geq 1}\right\rangle=0 .
$$

Therefore, by (2.36), we have

$$
\langle\Psi \mid \Psi\rangle=\left\langle\Phi_{0}, \Phi_{0}\right\rangle \geq 0 .
$$

Remark 2.3. By the above theorem, we observe that, if $\rho \in H^{-1 / 2}\left(\mathbb{R}^{3}\right) \cap$ $H^{-3 / 2}\left(\mathbb{R}^{3}\right)$, then $\mathcal{V}_{\text {phys }}$ is not trivial, i.e. $\mathcal{V}_{\text {phys }} \neq\{0\}$.

Let $H_{\mathrm{f}, k}(k=2,3)$ be selfadjoint operators given by

$$
H_{\mathrm{f}, k}=\sum_{j \leq k} \int_{\mathbb{R}^{3}} \omega(\mathbf{k}) a_{j}^{*}(\mathbf{k}) a_{j}(\mathbf{k}) d \mathbf{k} .
$$

If $\rho \in H_{\omega}^{-1}\left(\mathbb{R}^{3}\right)$, we define a scalar quantity $E_{\rho} \in \mathbb{R}$ by

$$
E_{\rho}=\frac{1}{2} \int d \mathbf{k} \frac{|\hat{\rho}(\mathbf{k})|^{2}}{\omega(\mathbf{k})^{2}} .
$$


If $\rho \in H_{\omega}^{-3 / 2}\left(\mathbb{R}^{3}\right)$, we define a non-zero vector $\Psi_{\rho}$ by

$$
\Psi_{\rho}=C_{\rho} \sum_{n=0}^{\infty} \frac{a_{0}^{*}\left(\frac{\hat{\rho}}{\omega^{3 / 2}}\right)^{n} \Omega}{2^{n / 2} n !},
$$

where $C_{\rho}$ is the normalized constant and

$$
C_{\rho}=\exp \left(-\frac{1}{2} \int_{\mathbb{R}^{3}} \frac{|\hat{\rho}(\mathbf{k})|^{2}}{\omega(\mathbf{k})^{3}} d \mathbf{k}\right) .
$$

Theorem 2.15. Let $\rho \in H_{\omega}^{-1 / 2}\left(\mathbb{R}^{3}\right) \cap H_{\omega}^{-3 / 2}\left(\mathbb{R}^{3}\right)$. For all $\Psi \in \mathcal{V}_{\text {phys }} \cap D(H)$, $H \Psi \in \mathcal{V}_{\text {phys }}$ and, for $\Psi^{\prime} \in \mathcal{V}_{\text {phys }}$,

$$
\left\langle\Psi^{\prime} \mid H \Psi\right\rangle=\left\langle\Psi^{\prime} \mid\left[H_{\mathrm{f}, 2}+E_{\rho}\right] \Psi\right\rangle .
$$

The vector $\Psi_{\rho}$ is an element of $\mathcal{V}_{\text {phys }}$ and an eigenvalue of $H$ corresponding to an eigenvalue $E_{\rho}$ :

$$
H \Psi_{\rho}=E_{\rho} \Psi_{\rho}
$$

Proof. We observe that

$$
\begin{aligned}
U_{\rho} H U_{\rho}^{*} & =W e^{-i \phi_{3}\left(i \hat{\rho} / \omega^{3 / 2}\right)}\left[H_{\mathrm{f}}+H_{\mathrm{int}}\right] e^{i \phi_{3}\left(i \hat{\rho} / \omega^{3 / 2}\right)} W \\
& =W\left[e^{-i \phi_{3}\left(i \hat{\rho} / \omega^{3 / 2}\right)} H_{\mathrm{f}} e^{i \phi_{3}\left(i \hat{\rho} / \omega^{3 / 2}\right)}+H_{\mathrm{int}}\right] W \\
& =W\left\{H_{\mathrm{f}}-\phi_{3}\left(\frac{\hat{\rho}}{\sqrt{\omega}}\right)+E_{\rho}+H_{\mathrm{int}}\right\} W
\end{aligned}
$$

By (2.31), we have

$$
\begin{gathered}
W H_{\mathrm{f}} W=H_{\mathrm{f}} \\
W \phi_{3}\left(\frac{\hat{\rho}}{\sqrt{\omega}}\right) W=\frac{1}{\sqrt{2}}\left[\phi_{3}\left(\frac{\hat{\rho}}{\sqrt{\omega}}\right)+\phi_{0}\left(\frac{\hat{\rho}}{\sqrt{\omega}}\right)\right], \\
W H_{\mathrm{int}} W=\frac{1}{2}\left[a_{3}\left(\frac{\hat{\rho}}{\sqrt{\omega}}\right)-a_{0}\left(\frac{\hat{\rho}}{\sqrt{\omega}}\right)-a_{3}^{*}\left(\frac{\hat{\rho}}{\sqrt{\omega}}\right)+a_{0}^{*}\left(\frac{\hat{\rho}}{\sqrt{\omega}}\right)\right] .
\end{gathered}
$$

Hence we obtain

$$
U_{\rho} H U_{\rho}^{*}=H_{\mathrm{f}}-a_{0}\left(\frac{\hat{\rho}}{\sqrt{\omega}}\right)-a_{3}^{*}\left(\frac{\hat{\rho}}{\sqrt{\omega}}\right)+E_{\rho} .
$$

Let $\Psi \in \mathcal{V}_{\text {phys }} \cap D(H)$. For the same reason as in the proof of Theorem 2.14, there exists a $\Phi \in \mathcal{F}_{3}$ such that $\Psi=U_{\rho}^{*} \Psi$ with

$$
\Phi=\Phi_{0}+\Phi_{\geq 1} \in \mathcal{F}_{2} \oplus\left[\mathcal{F}_{2}^{\perp} \cap \mathcal{F}_{3}\right] .
$$

Hence, by (2.43) and (2.44), we have

$$
\begin{aligned}
H \Psi & =U_{\rho}^{*}\left[H_{\mathrm{f}}-a_{0}\left(\frac{\hat{\rho}}{\sqrt{\omega}}\right)-a_{3}^{*}\left(\frac{\hat{\rho}}{\sqrt{\omega}}\right)+E_{\rho}\right] \Phi \\
& =U_{\rho}^{*}\left[H_{\mathrm{f}, 3}-a_{3}^{*}\left(\frac{\hat{\rho}}{\sqrt{\omega}}\right)+E_{\rho}\right] \Phi .
\end{aligned}
$$


Since

$$
\left[H_{\mathrm{f}, 3}-a_{3}^{*}\left(\frac{\hat{\rho}}{\sqrt{\omega}}\right)+E_{\rho}\right] \Phi \in \mathcal{F}_{3},
$$

we obtain $H \Psi \in \mathcal{V}_{\text {phys }}$. On the other hand, we can write

$$
\left[H_{\mathrm{f}, 3}-a_{3}^{*}\left(\frac{\hat{\rho}}{\sqrt{\omega}}\right)+E_{\rho}\right] \Phi=\Xi_{0}+\Xi_{\geq 1},
$$

where

$$
\begin{aligned}
& \Xi_{0}=\left[H_{\mathrm{f}, 2}+E_{\rho}\right] \Phi_{0} \in \mathcal{F}_{2}, \\
& \Xi_{\geq 1}=\left[H_{\mathrm{f}, 3}-a_{3}^{*}\left(\frac{\hat{\rho}}{\sqrt{\omega}}\right)+E_{\rho}\right] \Phi_{\geq 1}-a_{3}^{*}\left(\frac{\hat{\rho}}{\sqrt{\omega}}\right) \Phi_{0} \in \mathcal{F}_{2}^{\perp} \cap \mathcal{F}_{3} .
\end{aligned}
$$

In the same way as in the proof of Theorem 2.14 , for all $\Psi^{\prime} \in \mathcal{V}_{\text {phys }}$,

$$
\left\langle\Psi^{\prime} \mid H \Psi\right\rangle=\left\langle\Psi^{\prime} \mid \Xi_{0}\right\rangle=\left\langle\Psi^{\prime} \mid\left[H_{\mathrm{f}, 2}+E_{\rho}\right] U_{\rho}^{*} \Phi_{0}\right\rangle .
$$

Since $\left[H_{\mathrm{f}, 2}+E_{\rho}\right] \Phi_{\geq 1} \in \mathcal{F}_{2}^{\perp} \cap \mathcal{F}_{3}$ is commuting with $U_{\rho}^{*}$, we have

$$
\left\langle\Psi^{\prime} \mid\left[H_{\mathrm{f}, 2}+E_{\rho}\right] U_{\rho}^{*} \Phi_{\geq 1}\right\rangle=\left\langle\Psi^{\prime} \mid U_{\rho}^{*} \Phi_{\geq 1}\left[H_{\mathrm{f}, 2}+E_{\rho}\right]\right\rangle=0,
$$

which, combined with the above equation, implies the equation (2.40).

The equation (2.41) follows from direct calculation.

Theorem 2.16. (infrared divergence) Let $\rho \in H_{\omega}^{-1}\left(\mathbb{R}^{3}\right) \cap H_{\omega}^{-1 / 2}\left(\mathbb{R}^{3}\right)$. If $\rho \notin$ $H_{\omega}^{-3 / 2}\left(\mathbb{R}^{3}\right)$, then

$$
\mathcal{V}_{\text {phys }}=\{0\}
$$

Proof. Let us assume that there exists a physical state $\Psi=\in \mathcal{V}_{\text {phys }} \backslash 0$. Then there exists a positive integer $n_{0} \in \mathbb{N}$ such that $\Psi^{\left(n_{0}\right)} \neq 0$ and, for all $u \in D$,

$$
\left\langle\Psi^{\left(n_{0}\right)}, a_{\oplus^{4} L^{2}\left(\mathbb{R}^{3}\right)}(u, 0,0,-u) \Psi\right\rangle=C_{n_{0}} \int_{\mathbb{R}^{3}} \frac{u^{*}(\mathbf{k}) g(\mathbf{k})}{\omega(\mathbf{k})} d \mathbf{k},
$$

where we set

$$
C_{n_{0}}:=\frac{1}{\sqrt{2}} \int_{\mathbb{R}^{3 n_{0}}} \prod_{j=1}^{n_{0}} d \mathbf{k}_{j} \sum_{\mu_{1}, \cdots, \mu_{n_{0}}}\left|\Psi_{\mu_{1}, \cdots, \mu_{n_{0}}}^{\left(n_{0}\right)}\left(\mathbf{k}_{1}, \cdots, \mathbf{k}_{n_{0}}\right)\right|^{2}<\infty .
$$

By (2.11), we have, for all $u \in D$

$$
\begin{aligned}
\left|\int_{\mathbb{R}^{3}} \frac{u^{*}(\mathbf{k}) g(\mathbf{k})}{\omega(\mathbf{k})} d \mathbf{k}\right| & \leq \frac{1}{C_{n_{0}}}\left|\left\langle a_{\oplus^{4} L^{2}\left(\mathbb{R}^{3}\right)}^{*}(u, 0,0,-u) \Psi^{\left(n_{0}\right)}, \Psi\right\rangle\right| \\
& \leq \frac{\|\Psi\|}{C_{n_{0}}}\left\|a_{\oplus^{4} L^{2}\left(\mathbb{R}^{3}\right)}^{*}(u, 0,0,-u) \Psi^{\left(n_{0}\right)}\right\| \\
& \leq \frac{\sqrt{2}\|\Psi\|}{C_{n_{0}}} \int_{\mathbb{R}^{3}}|u(\mathbf{k})|^{2} d \mathbf{k}\left\|\left(N_{\mathrm{f}}+1\right)^{1 / 2} \Psi^{\left(n_{0}\right)}\right\| \\
& \leq \frac{\sqrt{2\left(n_{0}+1\right)}\|\Psi\|\left\|\Psi^{\left(n_{0}\right)}\right\|}{C_{n_{0}}} \int_{\mathbb{R}^{3}}|u(\mathbf{k})|^{2} d \mathbf{k} .
\end{aligned}
$$


Hence, by the density of $D$, there exists a bounded linear functional $L$ on $L^{2}\left(\mathbb{R}^{3}\right)$ such that

$$
L(u)=\int_{\mathbb{R}^{3}} \frac{g(\mathbf{k})^{*} u(\mathbf{k})}{\omega(\mathbf{k})} d \mathbf{k}, \quad u \in D .
$$

By Riesz representation theorem, there exists a vector $g \in L^{2}\left(\mathbb{R}^{3}\right)$ such that

$$
L(f)=\int_{\mathbb{R}^{3}} g(\mathbf{k})^{*} f(\mathbf{k}) d \mathbf{k}, \quad f \in L^{2}\left(\mathbb{R}^{3}\right) .
$$

In particular,

$$
\int_{\mathbb{R}^{3}} \frac{g(\mathbf{k})^{*} u(\mathbf{k})}{\omega(\mathbf{k})} d \mathbf{k}=\int_{\mathbb{R}^{3}} g(\mathbf{k}) u(\mathbf{k}) d \mathbf{k}, \quad u \in D .
$$

Let $f:=\hat{v} \times \check{w} \in \mathscr{S}\left(\mathbb{R}^{4}\right)$ with $v \in C_{0}^{\infty}(\mathbb{R})$ and $w \in C_{0}^{\infty}\left(\mathbb{R}^{3}\right)$ and set

$$
u:=\sqrt{\omega} F(f)=\sqrt{\omega} v(\omega) w \in D .
$$

By (2.45), we have

$$
\int_{\mathbb{R}^{3}}\left(\frac{\hat{\rho}(\mathbf{k})^{*}}{\omega(\mathbf{k})}-\sqrt{\omega(\mathbf{k})} g(\mathbf{k})\right) v(\omega(\mathbf{k})) w(\mathbf{k}) d \mathbf{k}=0 .
$$

Using du Bois-Reymond lemma, we observe that

$$
g^{*}=\frac{\hat{\rho}}{\omega^{3 / 2}} \in L^{2}\left(\mathbb{R}^{3}\right),
$$

which contradicts the condition $\rho \notin H_{\omega}^{-3 / 2}\left(\mathbb{R}^{3}\right)$.

\subsection{Example of the electrostatic field}

We consider a distribution density $\rho$ of charged particles with charge $e$ and positions $\boldsymbol{\xi}_{n} \in \mathbb{R}^{3}$ :

$$
\rho(\mathbf{x})=e \sum_{n=1}^{N} \delta\left(\mathbf{x}-\boldsymbol{\xi}_{n}\right), \quad \boldsymbol{\xi}_{n} \neq \boldsymbol{\xi}_{m}(n \neq m) .
$$

The Fourier transform of $\rho$ is of the form

$$
\hat{\rho}(\mathbf{k})=\frac{e}{(2 \pi)^{3 / 2}} \sum_{n=1}^{N} e^{-i \mathbf{k} \cdot \boldsymbol{\xi}_{n}} .
$$

For $\sigma, \kappa>0$, we set

$$
\rho_{\sigma, \kappa}(\mathbf{x})=\frac{1}{(2 \pi)^{3 / 2}} \int_{\sigma \leq|\mathbf{k}| \leq \kappa} \hat{\rho}(\mathbf{k}) e^{i \mathbf{k} \cdot \mathbf{x}} d \mathbf{k} .
$$

The Fourier transform of $\rho_{\sigma, \kappa}$ is given by

$$
\hat{\rho}_{\sigma, \kappa}(\mathbf{k})=\frac{e}{(2 \pi)^{3 / 2}} \sum_{n=1}^{N} I_{[\sigma, \kappa]}(\mathbf{k}) e^{-i \mathbf{k} \cdot \boldsymbol{\xi}_{n}},
$$


where $I_{[\sigma, \kappa]}$ is the indicator function of the set $\{\mathbf{k}|\sigma \leq| \mathbf{k} \mid \leq \kappa\}$. We observe that $\rho_{\sigma, \kappa}$ converges to $\rho$ :

$$
\lim _{\sigma \rightarrow 0, \kappa \rightarrow \infty} \rho_{\sigma, \kappa}=\rho \quad \text { in } \mathscr{S}^{\prime}\left(\mathbb{R}^{3}\right) .
$$

It is easy to see that

$$
\rho_{\sigma, \kappa} \in H_{\omega}^{s}\left(\mathbb{R}^{3}\right), \quad s \in \mathbb{R} .
$$

In particular, we have $\rho_{\sigma, \kappa} \in H^{-1 / 2}\left(\mathbb{R}^{3}\right) \cap H^{-3 / 2}\left(\mathbb{R}^{3}\right)$. We observe, by Theorem 2.14 and Remark 2.3, that the physical subspace $\mathcal{V}_{\text {phys }}$ is not trivial and, by Theorem 2.15, that the Hamiltonian $H$ has the expression

$$
\langle\Phi \mid H \Psi\rangle=\left\langle\Phi \mid\left[\sum_{j=1,2} \int_{\mathbb{R}^{3}} d \mathbf{k} \omega(\mathbf{k}) a_{j}^{*}(\mathbf{k}) a_{j}(\mathbf{k})+E_{\rho_{\sigma, \kappa}}\right] \Psi\right\rangle
$$

for all $\Phi \in \mathcal{V}_{\text {phys }}$ and $\Psi \in \mathcal{V}_{\text {phys }} \cap D\left(H_{f}^{2}\right)$. We can write

$$
E_{\rho_{\sigma, \kappa}}=\frac{1}{2} \int d \mathbf{k} \frac{\left|\widehat{\rho_{\sigma, \kappa}}(\mathbf{k})\right|^{2}}{\omega(\mathbf{k})^{2}}=E_{\sigma, \kappa}+V_{\sigma, \kappa},
$$

where

$$
E_{\sigma, \kappa}=\frac{e^{2} N}{2(2 \pi)^{3 / 2}} \int_{\sigma \leq|\mathbf{k}| \leq \kappa} \frac{1}{\omega(\mathbf{k})^{2}} d \mathbf{k}
$$

and

$$
V_{\sigma, \kappa}=\sum_{n \neq m} \frac{e^{2}}{2(2 \pi)^{3 / 2}} \int_{\sigma \leq|\mathbf{k}| \leq \kappa} \frac{e^{i \mathbf{k} \cdot\left(\boldsymbol{\xi}_{n}-\boldsymbol{\xi}_{m}\right)}}{\omega(\mathbf{k})^{2}} d \mathbf{k} .
$$

It is easy to see that

$$
\lim _{\sigma \rightarrow 0, \kappa \rightarrow \infty} E_{\sigma, \kappa}=\infty, \quad \lim _{\sigma \rightarrow 0, \kappa \rightarrow \infty} V_{\sigma, \kappa}=\sum_{n<m} \frac{e^{2}}{4 \pi\left|\boldsymbol{\xi}_{n}-\boldsymbol{\xi}_{m}\right|}
$$

(see [1] for details). Hence we have

$$
\lim _{\sigma \rightarrow 0, \kappa \rightarrow \infty}\left\langle\Phi \mid\left[H-E_{\sigma, \kappa}\right] \Psi\right\rangle=\left\langle\Phi \mid\left[\sum_{j=1,2} \int_{\mathbb{R}^{3}} d \mathbf{k} \omega(\mathbf{k}) a_{j}^{*}(\mathbf{k}) a_{j}(\mathbf{k})+\sum_{n<m} \frac{e^{2}}{4 \pi\left|\boldsymbol{\xi}_{n}-\boldsymbol{\xi}_{m}\right|}\right] \Psi\right\rangle
$$

for all $\Phi \in \mathcal{V}_{\text {phys }}$ and $\Psi \in \mathcal{V}_{\text {phys }} \cap D\left(H_{f}^{2}\right)$. The first term of the right hand side in the above equation is the Hamiltonian of the transverse wave of the photon and the second term is the Coulomb interaction between the charged particles.

Let us next consider the case where the distribution $\rho_{\kappa}$ given by

$$
\rho_{\kappa}(\mathbf{x})=\frac{1}{(2 \pi)^{3 / 2}} \int_{|\mathbf{k}| \leq \kappa} \hat{\rho}(\mathbf{k}) e^{i \mathbf{k} \cdot \mathbf{x}} d \mathbf{k},
$$

which is infrared singular : $\rho_{\kappa} \notin H_{\omega}^{-3 / 2}\left(\mathbb{R}^{3}\right)$. Indeed, since $|\hat{\rho}(0)|^{2}=(2 \pi)^{-3} e^{2} N^{2}>$ 0 , for sufficiently small $0<\delta<\kappa$,

$$
\int_{\mathbb{R}^{3}} \frac{\left|\hat{\rho_{\kappa}}(\mathbf{k})\right|^{2}}{\omega(\mathbf{k})^{3}}=\int_{|\mathbf{k}| \leq \kappa} \frac{|\hat{\rho}(\mathbf{k})|^{2}}{\omega(\mathbf{k})^{3}} \geq \int_{|\mathbf{k}| \leq \delta} \frac{C_{\delta}}{\omega(\mathbf{k})^{3}}=\infty,
$$


where $C_{\delta}:=\inf _{|\mathbf{k}| \leq \delta}\left|\hat{\rho}_{\kappa}(0)\right|^{2}>0$. On the other hand, we observe that, for all $\alpha<3 / 2$,

$$
\int_{\mathbb{R}^{3}} \frac{\left|\hat{\rho_{\kappa}}(\mathbf{k})\right|^{2}}{\omega(\mathbf{k})^{3}}=\int_{|\mathbf{k}| \leq \kappa} \frac{|\hat{\rho}(\mathbf{k})|^{2}}{\omega(\mathbf{k})^{3}} \leq \int_{|\mathbf{k}| \leq \kappa} \frac{e^{2} N^{2}}{(2 \pi)^{3} \omega(\mathbf{k})^{2 \alpha}}<\infty .
$$

In particular, we have $\rho \in H_{\omega}^{-1 / 2}\left(\mathbb{R}^{3}\right) \cap H_{\omega}^{-1}\left(\mathbb{R}^{3}\right)$. In this case, by Theorem 2.16 , the physical subspace is trivial.

\section{Non-Fock representation}

Assume that $\rho \notin H_{\omega}^{-3 / 2}\left(\mathbb{R}^{3}\right)$ but that $\rho \in H_{\omega}^{-1}\left(\mathbb{R}^{3}\right) \cap \in H_{\omega}^{-1 / 2}\left(\mathbb{R}^{3}\right)$. In this section, by using the non-Fock representation, we construct the quantized radiation field interacting with the classical source $\rho$ such that the physical subspace is not trivial.

Let

$$
a_{\mu}^{L}(f)=a_{\mu}(f)+\frac{\delta_{\mu 0}}{\sqrt{2}} \int_{\mathbb{R}^{3}} \frac{f^{*}(\mathbf{k}) \hat{\rho}(\mathbf{k})}{\omega(\mathbf{k})^{3 / 2}} d \mathbf{k}, \quad f \in D\left(\omega^{-1 / 2}\right),
$$

which obeys the CCRs on $\mathcal{F}_{0}$ : for all $f, g \in D\left(\omega^{-1 / 2}\right)$,

$$
\begin{gathered}
{\left[a_{\mu}^{L}(f), a_{\nu}^{L^{\dagger}}(g)\right]=-g_{\mu \nu} \int_{\mathbb{R}^{3}} f^{*}(\mathbf{k}) g(\mathbf{k}) d \mathbf{k},} \\
{\left[a_{\mu}^{L}(f), a_{\nu}^{L}(g)\right]=\left[a_{\mu}^{L^{\dagger}}(f), a_{\nu}^{L^{\dagger}}(g)\right]=0 .}
\end{gathered}
$$

It is well known and easy to see, from $\rho \notin H^{-3 / 2}\left(\mathbb{R}^{3}\right)$, that the transformation

$$
a_{\mu}(f) \longrightarrow a_{\mu}^{L}(f), \quad a_{\mu}^{\dagger}(f) \longrightarrow a_{\mu}^{L^{\dagger}}(f)
$$

is not proper (see [1] for details), i.e. there is no unitary operator $U$ such that $a_{\mu}^{L}(f)=U a_{\mu}(f) U^{*}$ and $a_{\mu}^{L^{\dagger}}(f)=U a_{\mu}^{\dagger}(f) U^{*}$. Hence, the representation $\left\{a_{\mu}^{L}(f) \mid f \in D\left(\omega^{-1 / 2}\right\}\right.$ of the CCRs is not equivalent to the representation $\left\{a_{\mu}(f) \mid f \in D\left(\omega^{-1 / 2}\right\}\right.$.

Let us construct the solution of the radiation operators by using the representation $\left\{a_{\mu}^{L}(f) \mid f \in D\left(\omega^{-1 / 2}\right\}\right.$ of the CCRs. For all $t \in \mathbb{R}$ and $f \in \mathscr{S}\left(\mathbb{R}^{3}\right)$, we set

$$
\begin{aligned}
& \boldsymbol{A}_{j}^{L}(t, f):=\frac{1}{\sqrt{2}} \sum_{\lambda=1}^{3}\left[a_{\lambda}\left(e_{j}^{(\lambda)} \frac{e^{i t \omega} \widehat{f^{*}}}{\sqrt{\omega}}\right)+a_{\lambda}^{\dagger}\left(e_{j}^{(\lambda)} \frac{e^{i t \omega} \hat{f}}{\sqrt{\omega}}\right)\right], \quad j=1,2,3, \\
& \boldsymbol{A}_{0}^{L}(t, f):=\frac{1}{\sqrt{2}}\left[a_{0}\left(\frac{e^{i t \omega} \widehat{f^{*}}}{\sqrt{\omega}}\right)+L\left(f^{*}\right)+a_{0}^{\dagger}\left(\frac{e^{i t \omega} \hat{f}}{\sqrt{\omega}}\right)+L(f)^{*}\right],
\end{aligned}
$$

where

$$
L(f)=\frac{1}{\sqrt{2}} \int_{\mathbb{R}^{3}} \frac{\hat{f}(\mathbf{k})^{*} \hat{\rho}(\mathbf{k})}{\omega(\mathbf{k})^{2}} d \mathbf{k} .
$$


We observe that, for all $f \in \mathscr{S}\left(\mathbb{R}^{3}\right)$,

$$
\begin{aligned}
& \boldsymbol{A}_{j}^{L}(0, f)=\frac{1}{\sqrt{2}} \sum_{\lambda=1}^{3}\left[a_{\lambda}^{L}\left(e_{j}^{(\lambda)} \frac{\widehat{f^{*}}}{\sqrt{\omega}}\right)+a_{\lambda}^{L^{\dagger}}\left(e_{j}^{(\lambda)} \frac{\hat{f}}{\sqrt{\omega}}\right)\right], \quad j=1,2,3, \\
& \boldsymbol{A}_{0}^{L}(0, f)=\frac{1}{\sqrt{2}}\left[a_{0}^{L}\left(\frac{\widehat{f^{*}}}{\sqrt{\omega}}\right)+a_{0}^{L^{\dagger}}\left(\frac{\hat{f}}{\sqrt{\omega}}\right)\right] .
\end{aligned}
$$

Hence,

$$
\boldsymbol{A}_{\mu}(0, f) \neq \boldsymbol{A}_{\mu}^{L}(0, f) .
$$

For $n \geq 1$ we set

$$
\begin{aligned}
& \boldsymbol{A}_{j}^{L^{(n)}}(t, f):=\frac{1}{\sqrt{2}} \sum_{\lambda=1}^{3}\left[a_{\lambda}\left(e_{j}^{(\lambda)} \frac{(i \omega)^{n} e^{i t \omega} \widehat{f^{*}}}{\sqrt{\omega}}\right)+a_{\lambda}^{\dagger}\left(e_{j}^{(\lambda)} \frac{(i \omega)^{n} e^{i t \omega} \hat{f}}{\sqrt{\omega}}\right)\right], \quad j=1,2,3, \\
& \boldsymbol{A}_{0}^{L^{(n)}}(t, f) \Psi:=\frac{1}{\sqrt{2}}\left[a_{0}\left(\frac{(i \omega)^{n} e^{i t \omega} \widehat{f^{*}}}{\sqrt{\omega}}\right)+a_{0}^{\dagger}\left(\frac{(i \omega)^{n} e^{i t \omega} \hat{f}}{\sqrt{\omega}}\right)\right] .
\end{aligned}
$$

In particular, we write $A_{\mu}^{L^{(1)}}=\dot{A}_{\mu}^{L}$ and $A_{\mu}^{L^{(2)}}=\ddot{A}_{\mu}^{L}$. In the same way as in Section 2, we observe that the map $\mathscr{S}\left(\mathbb{R}^{3}\right) \ni f \mapsto \boldsymbol{A}_{\mu}^{L}(t, f)$ is an operatorvalued distribution on $\mathbb{R}^{3}$ acting on $\left(\mathcal{F}, D\left(N_{\mathrm{f}}^{1 / 2}\right)\right)$ and that, for all $f \in \mathscr{S}\left(\mathbb{R}^{3}\right)$ and $\Psi \in D\left(N_{\mathrm{f}}^{1 / 2}\right)$, the map $\mathbb{R} \ni t \mapsto \boldsymbol{A}_{\mu}^{L}(t, f) \Psi$ is infinitely differentiable and, for all $n \geq 1, t \in \mathbb{R}$ and $f \in \mathscr{S}\left(\mathbb{R}^{3}\right)$,

$$
\frac{d^{n}}{d t^{n}} \boldsymbol{A}_{\mu}^{L}(t, f) \Psi:=\boldsymbol{A}_{\mu}^{L^{(n)}}(t, f) \Psi
$$

It is easy to see that a similar consequence as Theorem 2.5 hold:

Theorem 3.1. (Heisenberg equations and field equations)

(i) (observable) For all real-valued functions $f \in \mathscr{S}\left(\mathbb{R}^{3}\right), \boldsymbol{A}_{\mu}^{L^{(n)}}(f)(n \geq 0)$ are essentially $\eta$-selfadjoint on $\mathcal{F}_{0}$.

(ii)(equal-time commutation relation) For all $f, g \in \mathscr{S}\left(\mathbb{R}^{3}\right)$,

$$
\begin{aligned}
& {\left[\boldsymbol{A}_{\mu}^{L}(t, f), \dot{\boldsymbol{A}}_{\nu}^{L}(t, g)\right]=i g_{\mu \nu} \int_{\mathbb{R}^{3}} f(\mathbf{k}) g(\mathbf{k}) d \mathbf{k},} \\
& {\left[\boldsymbol{A}_{\mu}^{L}(t, f), \boldsymbol{A}_{\nu}^{L}(t, g)\right]=\left[\dot{\boldsymbol{A}}_{\mu}^{L}(t, f), \dot{\boldsymbol{A}}_{\nu}^{L}(t, g)\right]=0, \text { on } \mathcal{F}_{0} .}
\end{aligned}
$$

(iii) (Heisenberg equations) For all $f \in \mathscr{S}\left(\mathbb{R}^{3}\right)$ and $\Psi \in \mathcal{F}_{0}$,

$$
\dot{\boldsymbol{A}}_{\mu}^{L}(t, f) \Psi=i\left[H^{L}, \boldsymbol{A}_{\mu}^{L}(t, f)\right] \Psi,
$$

where

$$
H^{L}=H_{\mathrm{f}}+E_{\rho} .
$$

(iv) (field equations) For all $f \in \mathscr{S}\left(\mathbb{R}^{3}\right)$

$$
\begin{aligned}
& \ddot{A}_{j}^{L}(t, f)=\boldsymbol{A}_{j}^{L}(t, \Delta f), \\
& \ddot{\boldsymbol{A}}_{0}^{L}(t, f)=\boldsymbol{A}_{0}^{L}(t, \Delta f)+\int_{\mathbb{R}^{3}} \rho(\mathbf{x}) f(\mathbf{x}) d \mathbf{x} .
\end{aligned}
$$


Hence we see that the operator-valued distribution $\mathscr{S}\left(\mathbb{R}^{3}\right) \ni f \mapsto \boldsymbol{A}_{\mu}^{L}(t, f)$ is the solution of the field equations (2.14) and (2.15) with the initial values (3.1) and (3.2). For the uniqueness of the solution, we also have similar consequences as Theorem 2.6 and Corollary 2.7. It is clear that the operator $H^{L}$ defined by (3.4) is $\eta$-selfadjoint. By the equation (3.3), we see that $H^{L}$ is the Hamiltonian of the radiation fields interacting with the classical source $\rho$.

Remark 3.1. Formally, the Hamiltonian $H^{L}$ in this representation is given by

$$
\begin{array}{r}
H^{L}=\int_{\mathbb{R}^{3}} \omega(\mathbf{k}) \sum_{j=1}^{3}\left[a_{j}^{L^{\dagger}}(\mathbf{k}) a_{j}^{L}(\mathbf{k})-a_{0}^{L^{\dagger}}(\mathbf{k}) a_{0}^{L}(\mathbf{k})\right] \\
+\int_{\mathbb{R}^{3}} \frac{\mathbf{d k}}{\sqrt{2|\mathbf{k}|}}\left[\hat{\rho}(\mathbf{k})^{*} a_{0}^{L}(\mathbf{k})+\hat{\rho}(\mathbf{k}) a_{0}^{L^{\dagger}}(\mathbf{k})\right],
\end{array}
$$

where we denote

$$
a_{\mu}^{L}(\mathbf{k})=a_{\mu}(\mathbf{k})+\frac{\delta_{\mu 0}}{\sqrt{2}} \frac{\hat{\rho}(\mathbf{k})}{\omega(\mathbf{k})^{3 / 2}}, \quad a_{\mu}^{L^{\dagger}}(\mathbf{k})=a_{\mu}^{\dagger}(\mathbf{k})+\frac{\delta_{\mu 0}}{\sqrt{2}} \frac{\hat{\rho}(\mathbf{k})^{*}}{\omega(\mathbf{k})^{3 / 2}} .
$$

Hence, by direct calculation, we see that the expression (3.5) of $H^{L}$ is formally equal to (3.4).

Similarly as Proposition 2.8, for all $f \in \mathscr{S}\left(\mathbb{R}^{4}\right)$, there exists a unique operator $\mathbf{A}_{\mu}^{L}(f)$ such that, for all $h \in \mathscr{S}(\mathbb{R})$ and $g \in \mathscr{S}\left(\mathbb{R}^{3}\right)$,

$$
\boldsymbol{A}_{\mu}^{L}(h \times g) \Psi=\int_{\mathbb{R}} h(t) \boldsymbol{A}_{\mu}^{L}(t, g) \Psi d t, \quad h \in \mathscr{S}(\mathbb{R}), g \in \mathscr{S}\left(\mathbb{R}^{3}\right), \quad \Psi \in D\left(N_{\mathrm{f}}^{1 / 2}\right) .
$$

For all $f \in \mathscr{S}\left(\mathbb{R}^{4}\right)$ and $\Psi \in D\left(N_{\mathrm{f}}^{1 / 2}\right)$, the field equation hold:

$$
\boldsymbol{A}_{j}^{L}(\square f) \Psi=0, \quad \boldsymbol{A}_{j}^{L}(\square f) \Psi=\int_{\mathbb{R}^{4}} \rho(\mathbf{x}) f(\mathbf{x}) d \mathbf{x} \Psi .
$$

Let us define the physical subspace $\mathcal{V}_{\text {phys }}^{L}$ in this representation by

$$
\mathcal{V}_{\text {phys }}^{L}=\left\{\Psi \in \mathcal{F} \mid\left[\partial^{\mu} \boldsymbol{A}_{\mu}^{L}(f)\right]^{(+)} \Psi=0\right\} .
$$

Theorem 3.2. Let $\rho \in H_{\omega}^{-1 / 2}\left(\mathbb{R}^{3}\right) \cap H_{\omega}^{-1}\left(\mathbb{R}^{3}\right)$. Then $\mathcal{V}_{\text {phys }}^{L}$ is non-negative and

$$
\mathcal{V}_{\text {phys }}^{L}=W \mathcal{F}_{3}
$$

Proof. Similar as in Theorem 2.14.

Remark 3.2. By the above theorem, the physical subspace $\mathcal{V}_{\mathrm{phys}}^{L}$ is not trivial.

Acknowledgments The author would like to thank Professor A. Arai for useful comments. 


\section{References}

[1] A. Arai, Fock spaces and quantum fields, Nippon-Hyouronsya, Tokyo (2000) (in Japanese).

[2] M. Babiker, The Lorentz gauge in non-relativistic quantum electrodynamics, Proc. R. Soc. Lond. A383 (1982), 485-502.

[3] K. Bleuler, Eine neue methode zur Behandlung der longitudinalen und skalaren photonen, Helv. Phys. Acta. 23 (1950), 567-586.

[4] S. N. Gupta, Theory of longitudinal photon in quantum electrodynamics, Proc. Phys. Soc. A63 (1950), 681-691.

[5] C. Itzykson and j.-B. Zuber, Quantum field Theory, McGraw-Hill, New York (1980)

[6] Kugo and Ojima, Local covariant operator formalism of non-abelian gauge theories and quark confinement problem, Prog. Theor. Phys. Suppl. 66 (1979), 1-130.

[7] K. L. Nagy, State spaces with indefinite metric in quantum field theory, P. Noordhoff, Groningen, Netherlands (1966).

[8] N. Nakanishi, Indefinite-metric quantum field theory, Prog. Theor. Phys. Suppl. 51 (1972), 1-95.

[9] M. E. Peskin and D. V. Schroeder, An introduction to quantum field theory, Westview Press, the United State of America (1995).

[10] S. S. Schweber, An introduction to relativistic quantum field theory, Harper and Row, Publishers, New-York, (1962).

[11] H. Spohn, Dynamics of charged particles and their radiation field, Cambridge University Press, United Kingdom (2004).

[12] S. Sunakawa, Quantum electrodynamics with the indefinite metric, Prog. Theor. Phys. 19 (1958), 221-237.

[13] A. S. Wightman and L. Gårding, Field as operator-valued distributions in quantum field theory, Ark. $\ddot{\text { fr Phys. }} 28$ (1964), 129-184. 